ESAIM: COCV 27 (2021) 23

https://doi.org/10.1051/cocv/2021025
ESAIM: Control, Optimisation and Calculus of Variations

www.esaim-cocv.org

\title{
DISCRETE-TO-CONTINUUM LIMITS OF PLANAR DISCLINATIONS
}

\author{
Pierluigi Cesana ${ }^{1, *}$ and Patrick van Meurs ${ }^{2}$
}

\begin{abstract}
In materials science, wedge disclinations are defects caused by angular mismatches in the crystallographic lattice. To describe such disclinations, we introduce an atomistic model in planar domains. This model is given by a nearest-neighbor-type energy for the atomic bonds with an additional term to penalize change in volume. We enforce the appearance of disclinations by means of a special boundary condition. Our main result is the discrete-to-continuum limit of this energy as the lattice size tends to zero. Our proof relies on energy relaxation methods. The main mathematical novelty of our proof is a density theorem for the special boundary condition. In addition to our limit theorem, we construct examples of planar disclinations as solutions to numerical minimization of the model and show that classical results for wedge disclinations are recovered by our analysis.
\end{abstract}

Mathematics Subject Classification. 74E15, 74Q05, 49J45, 35A15.

Received May 27, 2020. Accepted March 2, 2021.

\section{INTRODUCTION}

This paper is devoted to the mathematical analysis of a discrete model that describes frustrations in atomistic lattices induced by rotational mismatches. Such configurations are called wedge disclinations, which are angular defects. Disclinations are observed in solids in situations where the rotational symmetry is violated at the level of the crystal lattice. Figure 1 illustrates classical, simple examples of disclinations.

Historically, the existence of disclinations was predicted by Volterra alongside dislocations (translational defects) in a celebrated paper [44]. However, it was not until the late 1960s that disclinations saw a systematical investigation both from an experimental and theoretical perspective. First examples of disclinations over planar lattices have been discovered in superconductors and reported in [22, 43]. While a dislocation is a singularity of the deformation field which may be described by a lattice-valued vector, called Burgers vector, a disclination, as stated in [1] (see also [38]), is characterized by a closure failure of rotation ... for a closed circuit round the disclination centre. A continuum theory for disclinations in linearized elasticity has been derived by de Wit in [16] based on the idea of compatible elasticity and later elaborated by the same author in a series of articles [1719]. A comprehensive theory for disclinations (alongside dislocations) in non-linear elasticity has been developed by Zubov [46]. We refer to [34] for the investigation of wedge disclinations in the realm of the elastoplastic defect theory, [41] for a review of mechanical models of disclinations and [45] for more recent developments on unified approaches to treat dislocations and disclinations.

Keywords and phrases: Disclinations, discrete to continuum limits, non-linear elasticity, relaxation, Gamma-convergence.

${ }^{1}$ Institute of Mathematics for Industry, Kyushu University, 744 Motooka, Fukuoka 819-0395, Japan.

2 Faculty of Mathematics and Physics, Kanazawa University, Kanazawa, Ishikawa 920-1192, Japan.

* Corresponding author: pcesana81@gmail.com 

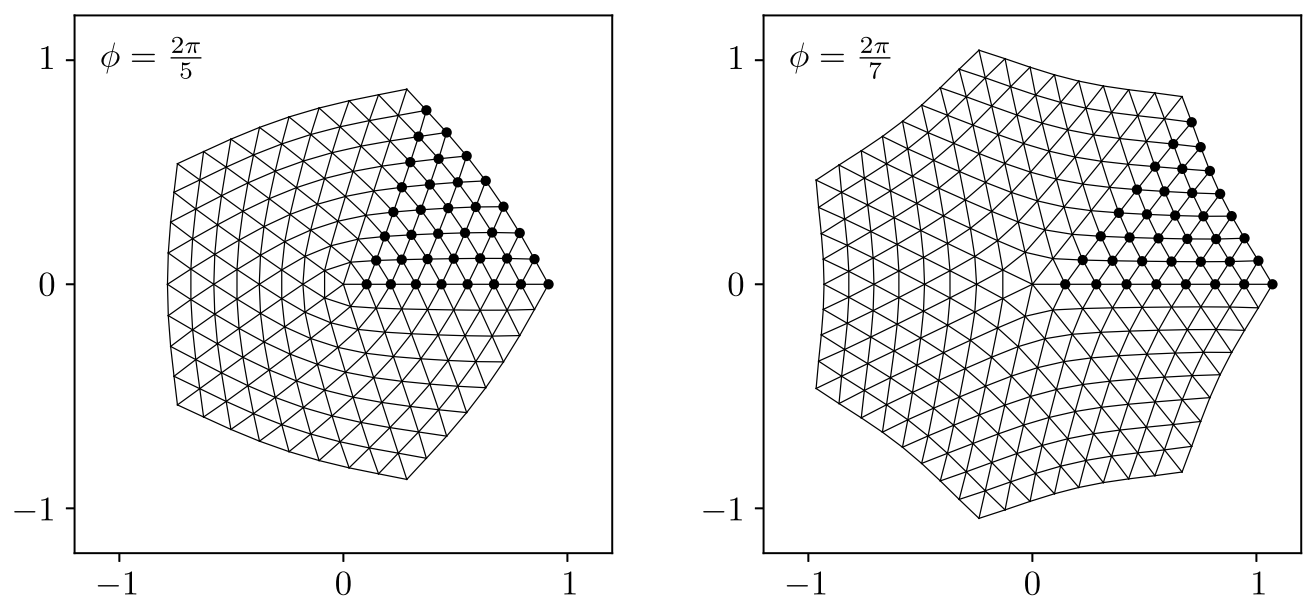

Figure 1. Simple examples of two wedge disclinations in planar triangular lattices. The left figure shows a 5 -type or positive disclination and the right figure a 7 -type or negative disclination. The atom in the center has a different number of bonds than the other atoms. $\phi$ is the angle between two such consecutive bonds. The lattice consists of rotated copies of the single wedge indicated by the thick dots.

Our work is inspired by a number of experimental observations which have revealed formation of disclinations in metallic structures under a variety of mechanical loading and forces, geometrical regimes and kinematical constraints. Here, we elaborate on two such observations.

In austenite-to-martensite transformations, disclinations may emerge during the formation of rotated (and constant-strain) shear-bands [6]. Such transformations are purely elastic and of the type solid-to-solid. They appear in a class of metals; in particular, in shape-memory alloys. Upon symmetry-break (typically triggered and driven by a negative temperature gradient), austenite, the high-symmetry and highly homogeneous crystal phase, turns into martensite, an anisotropic crystal phase with lower symmetry. The crystallographic lattice accommodates this phase change by forming a complicated microstructure composed of a mixture of thin plates and needle-shaped regions exhibiting differently rotated copies of martensitic phases. In a zero stressmicrostructure, martensite can be described by a piecewise constant deformation gradient. Constant-strain regions, that is, regions of constant crystal orientation, are separated by sharp planar interfaces according to kinematical compatibility. This compatibility is called a rank-1 connection of the corresponding deformation tensors. However, such configurations are ideal and typically atomistic non-idealities such as dislocations and disclinations appear in large numbers. Outstanding examples of disclinations in martensite are represented by "nested" star-shaped geometries observed in $\mathrm{Pb}_{3}\left(\mathrm{VO}_{4}\right)_{2}$. Experiments are described in [32, 37]; modeling work as well as numerical and exact constructions are described in $[11,39]$ respectively, and mathematical theories are developed in [9]. Significantly more complicated microstructures rich in defects are described in [30] for Ti-Nb-Al-based alloys. Here, martensite nucleates and evolves in the form of thin plates embedded in an austenitic lattice. The evolution of these plates is complex due to plates colliding against surrounding structures, undergoing further branching into additional martensitic sub-plates or being reflected after hitting a grain boundary. Such evolution results in self-similar patterns resembling fractal structure which are rich in disclinations and dislocations [30]. The available models of such microstructures are essentially based on statistical analysis [29] and probability [5, 10], and thus the consistency with atomistic models remains elusive. This is where we aim to contribute.

The second experimental observation is the discovery of a superior (and, yet to date, largely unexplained) structural reinforcement mechanism which has recently spurred scientific interest on the experimental as well as on the theoretical investigation of kink formation in certain classes of metal alloys. Formation of kinks consisting of approximately constant-strain bands accompanied by high rotational stretches of the lattice are observed in 
classes of laminate "mille-feuille" structures under uniaxial compression [25, 26]. In one of their most typical morphologies, bands manifest themselves in the form of planar and sharp ridge-shaped regions which appear at various length-scales and are accompanied by localized plastic stresses and formation of disclinations [36]. Kinks of various morphologies have been described in [31] where constructions of piecewise affine deformation maps based on the rank-1 connection rule and incorporating angular mismatches are presented. While [31]'s analysis sheds light on the kinematics of the disclination-kinking mechanism for various morphologies of kinks, there is no available model based on atomistic descriptions which describes the energy of such disclination-kinking mechanisms.

A common aspect on both martensitic microstructures and kink formation is that planar regions of approximately constant strains and constant-orientation lattices need to rotate in order to preserve the continuity of the deformation field across their common border. As a result, these materials necessarily develop angular lattice misfits which are striking examples of wedge disclinations. This motivates the modeling assumption of this paper that wedge disclinations are caused by large (non-linear) rotational stretches in planar-confined geometries.

Our aim is to take the first step in the direction of a comprehensive variational theory that is suitable to simultaneously treat microscale and localized defects and to predict their effect on large (non-linear) elastic and plastic deformations including kinks and shear-bands. By designing a simple, nearest-neighbor-type interaction mechanism, we construct a model which we apply to describe a single disclination in a planar lattice and which is at the same time potentially adaptable to describe multi-disclination systems and to incorporate other lattice defects such as voids, dislocations and grain boundaries.

By pursuing this aim, we also fill a gap in the literature on atomistic modeling of planar lattice defects and the limit thereof as the lattice spacing tends to 0 . To identify this gap, we review related literature. First, in the framework of linearized elasticity, the formal asymptotic expansions in [42] provide a continuum model for lattice defects. Second, in [35] two triangular lattices with different lattice spacings are attached together, which forces dislocations to form at the interface. The main result is a continuum limit of this model as the lattice spacing tends to 0 . Third, based on the discrete calculus of lattices constructed in [3], the stress in a periodic crystal induced by parallel screw dislocations is computed in $[4,20,27,28,40]$. These results provide quantitative estimates between the displacement in the lattice and the displacement computed from linear elasticity in the continuum counter part. In [7] these techniques are extended to capture cracks. All these works deal with either localized or small deformation to the underlying two dimensional lattice, which is unfit for disclinations (see, e.g., the relatively large deformations in Fig. 1).

For large deformations, the recent work in [33] presents and anlyzes an atomistic model of a stretchable hexagonal lattice defined over a smooth manifold, in which the main result is the continuum limit in the form of $\Gamma$-convergence. Since the approach in [33] is designed to describe the energetics of highly distorted membranes, we follow a similar approach. However, the choice in [33] that the number of bonds per atom is always 6 makes the result not applicable to disclinations.

As in [33], we assign an energy $E_{\varepsilon}$ to a deformed lattice, where $\varepsilon$ is the lattice spacing. Two examples of such deformed lattices are illustrated by the dotted nodes in Figure 1. Given a deformed lattice, the energy $E_{\varepsilon}$ penalizes stretching and compression of the atomic bonds and changes in volume of the triangles formed by three neighboring atoms. To enforce the appearance of a 5- or 7-type disclination, we require the deformed lattice to satisfy a special boundary condition such that 5 or 7 rotated copies of it fit together such as in Figure 1.

Our main result, Theorem 3.1, is the exact derivation of $E$, the macroscale energy obtained in the limit as $\varepsilon \rightarrow 0$, that is,

$$
E=\Gamma-\lim _{\varepsilon \rightarrow 0} E_{\varepsilon}
$$

in a suitable topology.

From the mathematical perspective, the interest of our analysis lies in the treatment of the special boundary condition. Conceptually, this boundary conditions requires us to incorporate a pointwise constraint in the 


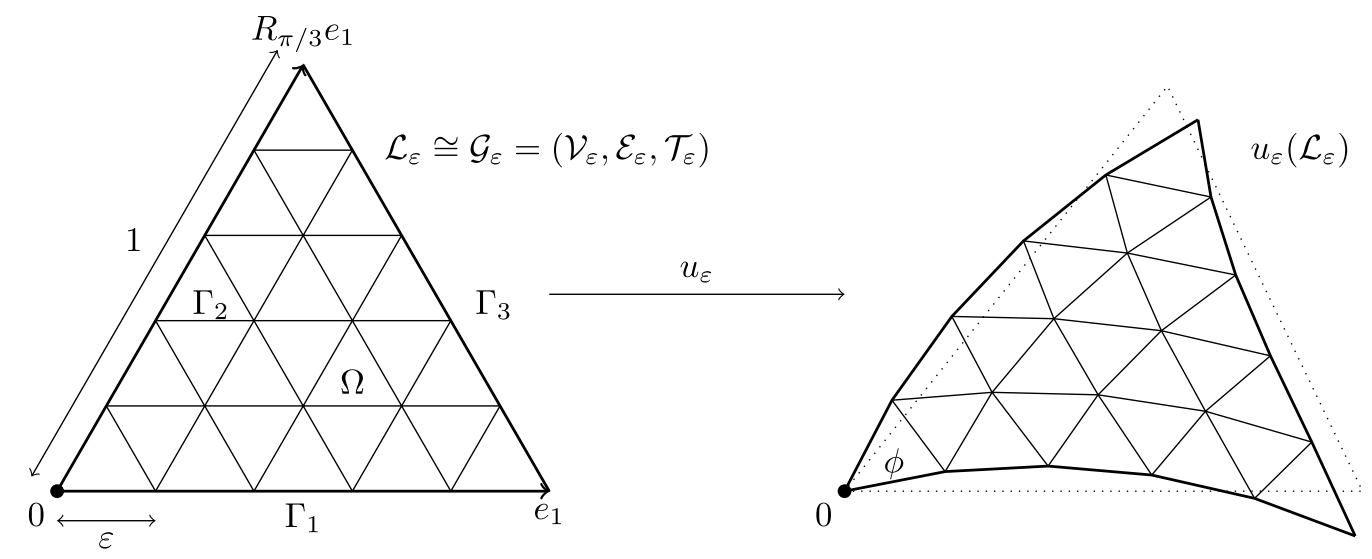

FiguRE 2. The reference lattice $\mathcal{L}_{\varepsilon}$ and an admissible deformation $u_{\varepsilon}$ (see (2.2)). The dotted triangle on the right is a visual aid to see that $u_{\varepsilon}$ satisfies the boundary condition.

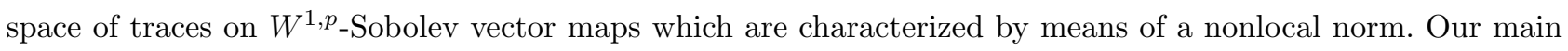
contribution on this is Proposition 4.4.

The paper is organized as follows. In Section 2 we present the discrete mechanical model and the mathematical setting required for the related variational analysis. In Section 3 we state and prove our main result, Theorem 3.1, on the $\Gamma$-convergence of the lattice energy $E_{\varepsilon}$. In this proof, we postpone the proofs of technical lemmas to Section 4, which includes the proof of Proposition 4.4 on density in the space of admissible lattice displacements. In Section 5 we explore the physical implications of our $\Gamma$-convergence analysis in terms of energy and stress states of the minimizers of the continuum model. Finally in Section 6 we present several numerical realizations of both positive and negative disclinations.

\section{The LATtice Energy $E_{\varepsilon}$}

Here we define the atomic lattice energy $E_{\varepsilon}$ as briefly introduced in the introduction. We start with the kinematics. Inspired by Figure 1, we consider a two-dimensional model. This corresponds conceptually to the mid-section of a 3-dimensional body. Furthermore, we impose rotational symmetry so that we may confine the domain to a single wedge in Figure 1 indicated by the black dots. The reference domain is given by the equilateral open triangle $\Omega \subset \mathbb{R}^{2}$ of size 1 with boundary $\Gamma=\cup_{i=1}^{3} \Gamma_{i}$ as depicted in Figure 2 . We take the reference positions of the atoms as a triangular lattice $\mathcal{L}_{\varepsilon}$, where $\mathcal{L}_{\varepsilon}$ is such that it fits on top of $\Omega$ as in Figure 2, i.e., the lattice spacing $\varepsilon \in(0,1)$ is such that $\frac{1}{\varepsilon} \in \mathbb{N}$ and $\mathcal{L}_{\varepsilon}$ is positioned such that each closed line segment $\Gamma_{i}$ fits on top of the atoms in one of the lattice directions. We denote by

$$
B^{\varepsilon}:=\left\{\varepsilon R_{\theta} e_{1}: \theta \in \frac{\pi}{3} \mathbb{Z}\right\}
$$

the set of the six outward-pointing bonds in $\mathcal{L}_{\varepsilon}$ from any lattice point, where $R_{\theta} \in S O(2)$ is the counter-clockwise rotation matrix by angle $\theta$. For later use, we sometimes interpret $\mathcal{L}_{\varepsilon}$ as a planar graph $\mathcal{G}_{\varepsilon}=\left(\mathcal{V}_{\varepsilon}, \mathcal{E}_{\varepsilon}, \mathcal{T}_{\varepsilon}\right)$, where $\mathcal{V}_{\varepsilon}$ is the set of all vertices $x_{i}, \mathcal{E}_{\varepsilon}$ is the set of all edges $e_{i j}$ between neighboring vertices $x_{i}, x_{j} \in \mathcal{V}_{\varepsilon}$, and $\mathcal{T}_{\varepsilon}$ is the set of all open triangles $T_{i j k} \in \Omega$ with sides given by the three edges $e_{i j}, e_{i k}, e_{j k} \in \mathcal{E}_{\varepsilon}$.

The set $\mathcal{A}_{\varepsilon}$ of admissible deformations is given by

$$
\mathcal{A}_{\varepsilon}=\left\{u_{\varepsilon}: \mathcal{L}_{\varepsilon} \rightarrow \mathbb{R}^{2}: u_{\varepsilon}\left(R_{\pi / 3} x\right)=R_{\phi} u_{\varepsilon}(x) \text { for all } x \in \Gamma_{1} \cap \mathcal{V}_{\varepsilon}\right\}
$$


where $\phi \in\left\{\frac{2 \pi}{5}, \frac{2 \pi}{7}\right\}$ is the angle associated with a 5- or 7-type disclination (see Fig. 1). Since $\mathcal{V}_{\varepsilon}$ is a finite set, the map $u_{\varepsilon}$ can be identified with a vector in $\mathbb{R}^{2\left|\mathcal{V}_{\varepsilon}\right|}$. The boundary condition in $(2.2)$ is such that the rotated copy $R_{\phi} u_{\varepsilon}\left(\mathcal{L}_{\varepsilon}\right)$ of $u_{\varepsilon}\left(\mathcal{L}_{\varepsilon}\right)$ fits seamlessly to $u_{\varepsilon}\left(\mathcal{L}_{\varepsilon}\right)$. Adding more rotated copies, we obtain a deformed lattice with rotational symmetry such as that in Figure 1, which has a 5 - or 7-type disclination at the origin.

Next we define the lattice energy on $\mathcal{A}_{\varepsilon}$ as inspired by [33]. We start with a formal description. Given $u_{\varepsilon} \in \mathcal{A}_{\varepsilon}$, we set the lattice energy formally as

$$
\tilde{E}_{\varepsilon}\left(u_{\varepsilon}\right)=\varepsilon^{2} \sum_{e_{i j} \in \mathcal{E}_{\varepsilon}} w\left(e_{i j}\right) \Phi\left(\frac{\left|u_{\varepsilon}\left(x_{i}\right)-u_{\varepsilon}\left(x_{j}\right)\right|}{\varepsilon}-1\right)+\frac{\varepsilon^{2}}{2} \sum_{T \in \mathcal{T}_{\varepsilon}} \Psi\left(\operatorname{sgn}\left(u_{\varepsilon}(T)\right) \frac{\left|u_{\varepsilon}(T)\right|}{|T|}\right),
$$

where the potential

$$
\Phi(r):=\frac{1}{p}|r|^{p}, \quad p \geq 2
$$

penalizes atomic bonds which are not of length $\varepsilon$ (the parameter value $p=2$ corresponds to linear elasticity), the weight function

$$
w: \mathcal{E}_{\varepsilon} \rightarrow\left\{\frac{1}{2}, 1\right\}, \quad w\left(e_{i j}\right)= \begin{cases}\frac{1}{2} & \text { if } e_{i j} \subset \Gamma \\ 1 & \text { otherwise }\end{cases}
$$

counts the outer edges as half, ${ }^{1}$ and the potential $\Psi$ penalizes change in volume of the triangles $T \in \mathcal{T}_{\varepsilon}$ (here, $|T|$ is the volume of $T$, and $\left|u_{\varepsilon}(T)\right|$ is the volume of the triangle $T$ after applying the displacement $u_{\varepsilon}$ ), especially if the volume of $T$ gets inverted under $u_{\varepsilon}$. Note that while the identity map minimizes $\tilde{E}_{\varepsilon}$, it is not in $\mathcal{A}_{\varepsilon}$ because of the boundary condition. Hence, the boundary condition enforces mechanical frustration. For $p=2$ and $\Psi \equiv 0$, Figure 1 illustrates the deformed lattice of a local minimizer in $\mathcal{A}_{\varepsilon}$ of $\tilde{E}_{\varepsilon}$ which does not contain negative change in volume. In this paper we always assume $p \geq 2$ unless specified otherwise.

Since the term involving $\Psi$ is not derived from physical principles, we elaborate on this modelling choice. It is well-known that when $\Psi \equiv 0$, lattice energies with only nearest-neighbor interactions such as $\tilde{E}_{\varepsilon}$ do not penalize folding or any other negative change in volume, and as a result, the related continuum energy may not penalize compression. While our boundary condition in (2.2) is not standard, we show in Section 6 by means of numerical simulations that folded patterns appear as local minimizers of $\tilde{E}_{\varepsilon}$ when $\Psi \equiv 0$.

To penalize folding, we pose the following minimal requirements on $\Psi$ :

( $\Psi 1) \exists C>0 \forall a, b \in \mathbb{R}:|\Psi(a)-\Psi(b)| \leq C\left(|a|^{\frac{p}{2}-1}+|b|^{\frac{p}{2}-1}+1\right)|a-b| ;$

$(\Psi 2) \quad \Psi \geq 0$, and $\Psi(a)=0 \Longleftrightarrow a=1$.

Condition $(\Psi 1)$ is a continuity estimate, which implies both local Lipschitz continuity and $\frac{p}{2}$-growth. Condition $(\Psi 2)$ ensures that any change in volume is penalized. A simple example of $\Psi$ is $\Psi(a)=|a-1|$. These conditions are more general than those in Section 3.3 of [33].

The term in $\tilde{E}_{\varepsilon}$ related to $\Psi$ has an inconvenient form. We fix this by changing variables. The result is a rigorously defined energy functional $E_{\varepsilon}$, which we will use in the remainder of the paper.

We change variables by describing the state $u_{\varepsilon}$ as the deformation of a displacement $U_{\varepsilon}$ defined on $\Omega$. Given $u_{\varepsilon} \in \mathcal{A}_{\varepsilon}$, we set $U_{\varepsilon}: \Omega \rightarrow \mathbb{R}^{2}$ as the piecewise linear extension of $u_{\varepsilon}$ to $\Omega$, i.e.,

$$
\begin{array}{r}
U_{\varepsilon}\left(\alpha x_{i}+\beta x_{j}+(1-\alpha-\beta) x_{k}\right):=\alpha u_{\varepsilon}\left(x_{i}\right)+\beta u_{\varepsilon}\left(x_{j}\right)+(1-\alpha-\beta) \\
\forall u_{\varepsilon}\left(x_{k}\right) \\
\forall T_{i j k} \in \mathcal{T}_{\varepsilon}, 0 \leq \alpha \leq 1,0 \leq \beta \leq 1-\alpha .
\end{array}
$$

\footnotetext{
${ }^{\mathrm{I}}$ We model edges as part of the volume of the medium around them.
} 
We note that

$$
\left[\nabla U_{\varepsilon}(x)\right]^{T} e=\frac{u_{\varepsilon}\left(x_{\ell}\right)-u_{\varepsilon}\left(x_{m}\right)}{\varepsilon} \quad \forall T_{i j k} \in \mathcal{T}_{\varepsilon}, x \in T_{i j k}
$$

where $e \in B^{1}$ is any lattice bond of unit direction (see (2.1)), and the indices $\ell, m \in\{i, j, k\}$ depend on $e$. In particular, $\nabla U_{\varepsilon}$ is constant on each $T \in \mathcal{T}_{\varepsilon}$,

$$
\left|\operatorname{det} \nabla U_{\varepsilon}\right|=\frac{\left|U_{\varepsilon}(T)\right|}{|T|} \text { on } T
$$

is the relative change in volume of $T$ under $U_{\varepsilon}$, and the sign of the determinant determines whether the volume of $T$ is inverted under $U_{\varepsilon}$. From these observations, we define the second term in (2.3) rigorously by

$$
\Psi\left(\operatorname{sgn}\left(u_{\varepsilon}(T)\right) \frac{\left|u_{\varepsilon}(T)\right|}{|T|}\right):=\Psi\left(\operatorname{det} \nabla U_{\varepsilon}\right) \quad \text { on } T
$$

Next we rewrite $\mathcal{A}_{\varepsilon}$ and $\tilde{E}_{\varepsilon}$ in terms of $U_{\varepsilon}$. This yields

$$
\mathcal{B}_{\varepsilon}^{\phi}:=\left\{U_{\varepsilon}: \bar{\Omega} \rightarrow \mathbb{R}^{2} \mid U_{\varepsilon} \text { is } \mathcal{L}_{\varepsilon} \text {-piecewise linear and } \forall x \in \Gamma_{1}: R_{\phi} U_{\varepsilon}(x)=U_{\varepsilon}\left(R_{\pi / 3} x\right)\right\} .
$$

Using (2.5) and (2.6), we rewrite (2.3) as

$$
\begin{aligned}
\frac{\sqrt{3}}{2} \tilde{E}_{\varepsilon}\left(u_{\varepsilon}\right)= & \frac{\sqrt{3}}{2}\left(\varepsilon^{2} \sum_{T \in \mathcal{T}_{\varepsilon}} \frac{1}{2} \sum_{e \in B^{1}} \frac{1}{|T|} \int_{T} \Phi\left(\left|e \nabla U_{\varepsilon}(x)\right|-1\right) \mathrm{d} x\right. \\
& \left.+\frac{\varepsilon^{2}}{2} \sum_{T \in \mathcal{T}_{\varepsilon}} \frac{1}{|T|} \int_{T} \Psi\left(\operatorname{det} \nabla U_{\varepsilon}(x)\right) \mathrm{d} x\right) \\
= & \int_{\Omega} \sum_{e \in B^{1}} \Phi\left(\left|e \nabla U_{\varepsilon}(x)\right|-1\right)+\Psi\left(\operatorname{det} \nabla U_{\varepsilon}(x)\right) \mathrm{d} x \\
= & \int_{\Omega} W\left(\nabla U_{\varepsilon}(x)\right) \mathrm{d} x,
\end{aligned}
$$

where

$$
W(A):=\sum_{e \in B^{1}} \Phi(|e A|-1)+\Psi(\operatorname{det} A) .
$$

In the computation above, the weight function $w\left(e_{i j}\right)$ turns into the factor $\frac{1}{2}$ due to the fact that each edge in the interior of $\Omega$ borders two triangles in $\mathcal{T}_{\varepsilon}$. Equation (2.7) motivates us to define

$$
E_{\varepsilon}: \mathcal{B}_{\varepsilon}^{\phi} \rightarrow[0, \infty), \quad E_{\varepsilon}\left(U_{\varepsilon}\right):=\int_{\Omega} W\left(\nabla U_{\varepsilon}(x)\right) \mathrm{d} x
$$

Remark 2.1 (Properties of $\left.\mathcal{B}_{\varepsilon}^{\phi}\right)$. We note that $\mathcal{B}_{\varepsilon}^{\phi} \subset \operatorname{Lip}\left(\bar{\Omega} ; \mathbb{R}^{2}\right)$ and that any $U_{\varepsilon} \in \mathcal{B}_{\varepsilon}^{\phi}$ satisfies $U_{\varepsilon}(0)=0$. While $\mathcal{B}_{\varepsilon}^{\phi}$ does not contain a subspace of constants, it does contain a subspace of linear maps $U_{\varepsilon}(x)=A x$. A possible choice for $A \in \mathbb{R}^{2 \times 2}$ is the one that satisfies $A e_{1}=e_{1}$ and $A\left(R_{\pi / 3} e_{1}\right)=R_{\phi} e_{1}$. 


\section{Continuum Limit}

Having introduced the lattice energy $E_{\varepsilon}$ on the triangular lattice, we are now in a position to discuss the continuum limit as $\varepsilon \rightarrow 0$. To keep track of the asymptotic behavior of minima and minimizers of $E_{\varepsilon}$ we characterize the continuum model with $\Gamma$-convergence [14].

Let $p \geq 2$. The domain of the continuum energy is

$$
W_{\phi}^{1, p}\left(\Omega ; \mathbb{R}^{2}\right):=\left\{U \in W^{1, p}\left(\Omega ; \mathbb{R}^{2}\right): R_{\phi} U_{\varepsilon}(x)=U_{\varepsilon}\left(R_{\pi / 3} x\right) \text { for a.e. } x \in \Gamma_{1}\right\} .
$$

Observe that $W_{\phi}^{1, p}\left(\Omega ; \mathbb{R}^{2}\right)$ is linear (and, in particular, convex), and non-empty since $\mathcal{B}_{\varepsilon}^{\phi} \cup W_{0}^{1, p}\left(\Omega ; \mathbb{R}^{2}\right) \subset$ $W_{\phi}^{1, p}\left(\Omega ; \mathbb{R}^{2}\right)$. Moreover, if $U \in W_{\phi}^{1, p}\left(\Omega ; \mathbb{R}^{2}\right)$, there holds $R U \in W_{\phi}^{1, p}\left(\Omega ; \mathbb{R}^{2}\right)$ for any $R \in S O(2)$. Thanks to the properties of traces (see Lem. 4.6), we have that $W_{\phi}^{1, p}\left(\Omega ; \mathbb{R}^{2}\right)$ is strongly closed and, therefore, weakly closed as well thanks to convexity.

The continuum energy functional is given by

$$
E: L^{p}\left(\Omega ; \mathbb{R}^{2}\right) \rightarrow[0, \infty], \quad E(U)= \begin{cases}\int_{\Omega} Q W(\nabla U) & U \in W_{\phi}^{1, p}\left(\Omega ; \mathbb{R}^{2}\right) \\ \infty & U \notin W_{\phi}^{1, p}\left(\Omega ; \mathbb{R}^{2}\right)\end{cases}
$$

where $W$ is as in (2.8) and $Q W$ is the quasiconvex envelop of $W$ defined by

$$
Q W(A):=\sup \{f(A): f \leq W, f \text { quasiconvex }\},
$$

where $f$ being quasiconvex means that $f: \mathbb{R}^{2 \times 2} \rightarrow \mathbb{R}$ is Borel measurable, locally bounded and satisfies

$$
f(A) \leq \frac{1}{|\omega|} \int_{\omega} f(A+\nabla \vartheta(x)) \mathrm{d} x
$$

for any bounded open set $\omega \subset \mathbb{R}^{2}$, any $A \in \mathbb{R}^{2 \times 2}$ and any $\vartheta \in W_{0}^{1, \infty}\left(\omega, \mathbb{R}^{2}\right)$.

Theorem 3.1. For $\phi \in\left\{\frac{2 \pi}{5}, \frac{2 \pi}{7}\right\}$ and $2 \leq p<\infty, E_{\varepsilon}$ (see (2.9)) $\Gamma$-converges as $\varepsilon \rightarrow 0$ to $E$ in the strong $L^{p}(\Omega)$ topology.

We prove Theorem 3.1 in Section 3.2. Since the $\varepsilon$-dependence of $E_{\varepsilon}$ appears only in its domain $\mathcal{B}_{\phi}^{\varepsilon}$, proving a $\Gamma$-limit result reduces to proving a relaxation result, i.e., finding the lower semi-continuous envelope of

$$
U \mapsto \int_{\Omega} W(\nabla U)
$$

in the right functional framework. There is a large literature on such relaxation problems; in Section 3.1 we cite the relevant classical theory. While this theory gives a useful roadmap for proving Theorem 3.1, it does not capture Theorem 3.1 because of the periodic boundary condition in $\mathcal{B}_{\varepsilon}^{\phi}$. Therefore, in Section 3.2 we give the skeleton of the proof of Theorem 3.1 based on the classical theory, and identify the missing steps as technical lemmas which we prove in Section 4.

\subsection{Classical relaxation result on $\mathcal{E}$}

We review some classical relaxation results as preparation for proving Theorem 3.1. All theorem references below in this section refer to Dacorogna's book [13]. Another relevant reference is [2]. 
We recall that $\Omega$ is a bounded Lipschitz domain. Here and in what follows we adopt the Frobenius norm for matrices. Let $\mathcal{W}: \mathbb{R}^{2 \times 2} \rightarrow \mathbb{R}$ satisfy

(W1) p-growth. $\exists C, C^{\prime}>0 \forall A \in \mathbb{R}^{2 \times 2}: \frac{1}{C}|A|^{p}-C \leq \mathcal{W}(A) \leq C^{\prime}\left(|A|^{p}+1\right)$;

(W2) Continuity estimate. $\exists C>0 \forall A, B \in \mathbb{R}^{2 \times 2}: \frac{|\mathcal{W}(A)-\mathcal{W}(B)|}{|A-B|} \leq C\left(|A|^{p-1}+|B|^{p-1}+1\right)$.

Note that $(W 1)$ includes a uniform bound from below, and that $(W 2)$ provides a local Lipschitz estimate. We set

$$
\mathcal{E}: L^{p}\left(\Omega ; \mathbb{R}^{2}\right) \rightarrow \mathbb{R}, \quad \mathcal{E}(U)= \begin{cases}\int_{\Omega} \mathcal{W}(\nabla U) & U \in W^{1, p}(\Omega) \\ \infty & U \notin W^{1, p}(\Omega) .\end{cases}
$$

Here and henceforth, we remove the range $\mathbb{R}^{2}$ from the notation of the function space if there is no danger for confusion.

As preparation, we cite Theorem 6.9 for the alternative characterization of (3.2) as the quasiconvexification of $\mathcal{W}$ given by

$$
Q \mathcal{W}(A)=\inf \left\{|\omega|^{-1} \int_{\omega} \mathcal{W}(A+\nabla \vartheta): \vartheta \in W_{0}^{1, \infty}\left(\omega, \mathbb{R}^{2}\right)\right\}
$$

where $\omega$ is a subset of $\mathbb{R}^{2}$ with $|\partial \omega|=0$ (i.e., $\partial \omega$ has zero two-dimensional volume).

Since $\mathcal{W}$ satisfies $(W 1)$, Theorem 9.1 implies that for all $U \in W^{1, p}(\Omega)$ there exists a sequence $\left(U_{k}\right)_{k} \subset$ $W_{0}^{1, p}(\Omega)+\{U\}$ such that

$$
U_{k} \rightarrow U \text { in } L^{p}(\Omega)
$$

and

$$
\mathcal{E}\left(U_{k}\right)=\int_{\Omega} \mathcal{W}\left(\nabla U_{k}(x)\right) \mathrm{d} x \rightarrow \int_{\Omega} Q \mathcal{W}(\nabla U(x)) \mathrm{d} x \quad \text { as } k \rightarrow \infty .
$$

By the two properties of $\mathcal{W}$, it follows from Theorem 6.9 and Theorem 5.3(iv) that $Q \mathcal{W}$ is continuous. Then, Theorem 1.13 implies that $U \mapsto \int Q \mathcal{W}(\nabla U)$ is sequentially weakly lower semicontinuous in $W^{1, p}(\Omega)$. In particular, for any $\left(U_{k}\right)_{k} \subset W^{1, p}(\Omega)$ converging weakly in $W^{1, p}(\Omega)$ to some $U$, we have that

$$
\liminf _{k \rightarrow \infty} \mathcal{E}\left(U_{k}\right) \geq \liminf _{k \rightarrow \infty} \int_{\Omega} Q \mathcal{W}\left(\nabla U_{k}(x)\right) \mathrm{d} x \geq \int_{\Omega} Q \mathcal{W}(\nabla U(x)) \mathrm{d} x .
$$

\subsection{Proof of Theorem 3.1}

The proof below relies on the following three statements which we make precise and prove in Section 4:

- Poincaré Inequality holds on $W_{\phi}^{1, p}(\Omega)$ (Lem. 4.1);

- $W$ satisfies Properties (W1) and (W2) defined in Section 3.1 (Lem. 4.2);

- $\mathcal{B}_{\phi}^{\varepsilon}$ is dense in $W_{\phi}^{1, p}(\Omega)$ (Prop. 4.4).

The proof of Theorem 3.1 follows from matching a lower bound with an upper bound, which is the standard method for computing $\Gamma$-limits [14]. To identify the set where the limit functional is finite, we first investigate the (equi-)compactness of minimizing sequences. 
Proof of Theorem 3.1. Compactness. By the lower bound in (W1),

$$
E_{\varepsilon}\left(U_{\varepsilon}\right) \geq \frac{1}{C}\left\|\nabla U_{\varepsilon}\right\|_{L^{p}(\Omega)}^{p}-C
$$

for some constants $C>0$ independent of $\varepsilon$ and $U_{\varepsilon}$, and thus any finite-energy sequence $\left(\nabla U_{\varepsilon}\right)_{\varepsilon}$ is bounded in $L^{p}(\Omega)$. By the Poincaré Inequality (Lem. 4.1), we then infer that $U_{\varepsilon}$ is bounded in $W^{1, p}(\Omega)$, and thus strongly convergent (along a subsequence) in $L^{p}(\Omega)$.

$\Gamma$-liminf. Since we can focus on finite-energy sequences $U_{\varepsilon} \rightarrow U$ in $L^{p}(\Omega)$, the compactness statement implies that $U_{\varepsilon} \rightarrow U$ in $W^{1, p}(\Omega)$ as $\varepsilon \rightarrow 0$. Since $\left(U_{\varepsilon}\right) \subset W_{\phi}^{1, p}(\Omega)$, which is a closed subspace of $W^{1, p}(\Omega)$, we also have $U \in W_{\phi}^{1, p}(\Omega)$. Hence, by applying (3.5), we obtain the required $\Gamma$-liminf estimate.

$\Gamma$-limsup. Thanks to (3.4) it suffices to find, for any $U \in W_{\phi}^{1, p}(\Omega)$, a sequence $\left(U_{\varepsilon}\right)_{\varepsilon} \subset \mathcal{B}_{\phi}^{\varepsilon}$ such that

$$
U_{\varepsilon} \stackrel{\varepsilon \rightarrow 0}{\longrightarrow} U \text { in } L^{p}(\Omega) \quad \text { and } \quad E_{\varepsilon}\left(U_{\varepsilon} \stackrel{\varepsilon \rightarrow 0}{\longrightarrow} \int_{\Omega} W(\nabla U) \mathrm{d} x=: \mathcal{E}(U)\right.
$$

To prove (3.6), we infer from Proposition 4.4 and $E_{\varepsilon}\left(U_{\varepsilon}\right)=\mathcal{E}\left(U_{\varepsilon}\right)$ that it is enough to show that $\mathcal{E}$ is continuous in $W^{1, p}(\Omega)$ at $U$. The continuity of $\mathcal{E}$ follows by $(W 2)$, Lemma 4.1 and Hölder's inequality from

$$
\begin{aligned}
|\mathcal{E}(U)-\mathcal{E}(V)| & \leq \int_{\Omega}|W(\nabla U)-W(\nabla V)| \mathrm{d} x \\
& \leq C \int_{\Omega}\left(|\nabla U|^{p-1}+|\nabla V|^{p-1}+1\right)|\nabla U-\nabla V| \mathrm{d} x \\
& \leq C\left(\|U\|_{W^{1, p}(\Omega)}^{p-1}+\|V\|_{W^{1, p}(\Omega)}^{p-1}+1\right)\|U-V\|_{W^{1, p}(\Omega)}
\end{aligned}
$$

where $V \in W^{1, p}(\Omega)$ is arbitrary.

\section{TeChnichl Steps IN THE PROOF OF TheOREM 3.1}

Here we state rigorously and prove the three statements mentioned at the start of Section 3.2. We start with the Poincaré Inequality.

Lemma 4.1 (Poincaré Inequality on $W_{\phi}^{1, p}(\Omega)$ ). There exists $C>0$ such that for all $U \in W_{\phi}^{1, p}(\Omega)$ it holds that

$$
\|U\|_{L^{p}(\Omega)} \leq C\|\nabla U\|_{L^{p}(\Omega)} .
$$

Proof. We follow a standard proof by contradiction. Assuming that there exists $\left(U_{n}\right) \subset W_{\phi}^{1, p}(\Omega)$ such that

$$
1=\left\|U_{n}\right\|_{L^{p}(\Omega)}>n\left\|\nabla U_{n}\right\|_{L^{p}(\Omega)},
$$

we obtain by compactness that $U_{n}$ converges along a subsequence (not relabelled) to $U$ strongly in $L^{p}(\Omega)$ and weakly in $W_{\phi}^{1, p}(\Omega)$. Hence, $\|U\|_{L^{p}(\Omega)}=1$ and $\|\nabla U\|_{L^{p}(\Omega)}=0$. Since $\Omega$ is connected, $U \equiv V_{0}$ for some constant and non-zero vector $V_{0} \in \mathbb{R}^{2}$. However, for a.e. $x \in \Gamma_{1}$,

$$
R_{\phi} U(x)-U\left(R_{\pi / 3} x\right)=\left(R_{\phi}-I\right) V_{0} \neq 0 .
$$

Hence, $U \notin W_{\phi}^{1, p}(\Omega)$, which completes the proof. 


\subsection{Properties of $W$}

Lemma 4.2. $W$ defined in (2.8) satisfies Properties (W1) and (W2) defined in Section 3.1.

Proof. If $\Psi \equiv 0$, then (W1) is obvious since $\Phi(r)=\frac{1}{p}|r|^{p}$. Then, since $\Psi \geq 0$ by ( $\left.\Psi 2\right)$, the lower bound in (W1) is immediate. The upper bound follows by $(\Psi 1)$ from

$$
\begin{aligned}
\Psi(\operatorname{det} A) & =\Psi(\operatorname{det} A)-\Psi(\operatorname{det} I) \\
& \leq C\left(|\operatorname{det} A|^{\frac{p}{2}-1}+2\right)|\operatorname{det} A-1| \\
& \leq C^{\prime}\left(|A|^{p-2}+2\right)\left(|A|^{2}+1\right) .
\end{aligned}
$$

$(W 2)$ follows by $(\Psi 1)$ from

$$
\begin{aligned}
|W(A)-W(B)| \\
\leq \sum_{e \in B^{1}}|\Phi(|e A|-1)-\Phi(|e B|-1)|+|\Psi(\operatorname{det} A)-\Psi(\operatorname{det} B)| \\
\leq \sum_{e \in B^{1}} C_{p}\left(|e A|^{p-1}+|e B|^{p-1}+1\right)|| e A|-| e B|| \\
\quad+C\left(|\operatorname{det} A|^{\frac{p}{2}-1}+|\operatorname{det} B|^{\frac{p}{2}-1}+1\right)|\operatorname{det} A-\operatorname{det} B| \\
\leq C^{\prime}\left(|A|^{p-1}+|B|^{p-1}+1\right)|A-B|+C^{\prime \prime}\left(|A|^{p-2}+|B|^{p-2}+1\right)(|A|+|B|)|A-B| \\
\leq C^{\prime \prime \prime}\left(|A|^{p-1}+|B|^{p-1}+1\right)|A-B| .
\end{aligned}
$$

For later use (although not necessary for the proof of Thm. 3.1) we elaborate on the rigidity properties of the energy density $W$. The rigidity estimate is a direct consequence of Assumptions (W1), (W2). Lengthy computations are postponed to the Appendix.

We start with introducing the singular value decomposition

$$
A=P_{1} \Sigma P_{2}, \quad \text { where } \Sigma:=\left[\begin{array}{cc}
\sigma_{1} & 0 \\
0 & \sigma_{2}
\end{array}\right] \text { and } P_{1}, P_{2} \in O(2) .
$$

In (4.1), $0 \leq \sigma_{1} \leq \sigma_{2}$ are the ordered singular values of $A$, that is, the square roots of the eigenvalues of the matrix $A A^{T}$. We also recall the definition of the distance function

$$
\operatorname{dist}^{p}(A, S O(2))=\inf _{Q \in S O(2)}|A-Q|^{p}
$$

Lemma 4.3. For $W$ defined in (2.8), there exists $c_{2}>0$ such that for all $A \in \mathbb{R}^{2 \times 2}$

$$
W(A) \geq c_{2} \operatorname{dist}^{p}(A, S O(2))
$$

Proof. Let $A=P_{1} \Sigma P_{2}$ be the singular decomposition as in (4.1). We split two cases; $\operatorname{det} A \geq 0$ and $\operatorname{det} A<0$. We start with the first case. From the definition of $W$ we get

$$
W(A) \geq \frac{1}{p} \sum_{e \in B_{1}}|| A e|-1|^{p}=\frac{1}{p} \sum_{e \in B_{1}}|| \Sigma P_{2} e|-1|^{p}=\frac{1}{p} \sum_{k=0}^{5}|| \Sigma R_{\theta+k \pi / 3} e_{1}|-1|^{p},
$$


where $e_{1}$ is the unit vector, $R$ is the rotation matrix, and $\theta \in[0, \pi / 3)$ is fixed by $P_{2}$. We first consider the case $p=2$. Thanks to Lemma A.1 we have

$$
14 \sum_{k=0}^{5}|| \Sigma R_{\theta+k \pi / 3} e_{1}|-1|^{2} \geq\left(\sigma_{1}-1\right)^{2}+\left(\sigma_{2}-1\right)^{2} .
$$

Recalling the well-known relation

$$
\left(\sigma_{1}-1\right)^{2}+\left(\sigma_{2}-1\right)^{2}=\operatorname{dist}^{2}(A, O(2))
$$

and noting that $\operatorname{det} A \geq 0$ implies $\operatorname{dist}(A, O(2))=\operatorname{dist}(A, S O(2))$, we obtain (4.2). For the case $p>2$, applying Jensen's inequality in (4.3) yields

$$
W(A) \geq \frac{6^{1-\frac{p}{2}}}{p}\left|\sum_{k=0}^{5}\left(\left|\Sigma R_{\theta+k \pi / 3} e_{1}\right|-1\right)^{2}\right|^{\frac{p}{2}} .
$$

Then, (4.2) follows from the argument above.

We continue with the second case, $\operatorname{det} A<0$. By (W1) in Section 3.1, there exist $M>0$ independent of $A$ such that $W(A) \geq \frac{1}{M}\left(|A|^{p}-M\right)$. We separate two cases:

1. If $|A| \leq 2 M$, then $\operatorname{det} A \geq-2 M^{2}$, and thus

$$
W(A) \geq \Psi(\operatorname{det} A) \geq \min _{\left[-2 M^{2}, 0\right]} \Psi
$$

which is positive by $(\Psi 2)$. On the other hand, for the right-hand side of (4.2), we obtain

$$
\operatorname{dist}^{p}(A, S O(2)) \leq \max _{|B| \leq 2 M} \operatorname{dist}^{p}(B, S O(2))<\infty
$$

Hence, there exists $c_{2}>0$ independent of $A$ such that (4.2) holds.

2. If $|A|>2 M$, we note from the triangle inequality

$$
\operatorname{dist}(A, S O(2)) \leq|A|+\operatorname{dist}(0, S O(2))=|A|+\sqrt{2}
$$

that

$$
W(A) \geq \frac{1}{M}|A|^{p}-M \geq c_{2}(|A|+\sqrt{2})^{p} \geq c_{2} \operatorname{dist}^{p}(A, S O(2))
$$

for some $c_{2}>0$ independent of $A$.

\subsection{Density of $\mathcal{B}_{\phi}^{\varepsilon}$ in $W_{\phi}^{1, p}(\Omega)$}

Proposition 4.4 (Density of $\mathcal{B}_{\phi}^{\varepsilon}$ in $W_{\phi}^{1, p}(\Omega)$ ). For all $U \in W_{\phi}^{1, p}(\Omega)$ there exists $U_{\varepsilon} \in B_{\phi}^{\varepsilon}$ parametrized by $\frac{1}{\varepsilon} \in \mathbb{N}$ such that $\left\|U-U_{\varepsilon}\right\|_{W^{1, p}(\Omega)} \rightarrow 0$ as $\varepsilon \rightarrow 0$.

Before giving the proof of Proposition 4.4 at the end of this section, we first outline the idea of the proof, and then establish some technical lemmas. 
In order to explain the idea of the proof, we first recall two classical density results in the following lemma. To state it, we define $\operatorname{Lip}_{\phi}(\bar{\Omega}):=\operatorname{Lip}(\bar{\Omega}) \cap W_{\phi}^{1, p}(\Omega)$.

Lemma 4.5 (Density of $\left.B_{\phi}^{\varepsilon}\right)$. For any $U: \Omega \rightarrow \mathbb{R}^{2}$ with either $U \in W_{0}^{1, p}(\Omega)$ or $U \in C^{1}(\Omega) \cap \operatorname{Lip}_{\phi}(\bar{\Omega})$ there exists $U_{\varepsilon} \in B_{\phi}^{\varepsilon}$ parametrized by $\frac{1}{\varepsilon} \in \mathbb{N}$ such that $\left\|U-U_{\varepsilon}\right\|_{W^{1, p}(\Omega)} \rightarrow 0$ as $\varepsilon \rightarrow 0$.

Proof. This is a standard result in numerical analysis; see e.g. [21, Chap. X, Prop. 2.1, 2.6 and 2.9]. We give the details of the proof to show how our boundary condition fits in.

For $U \in W_{0}^{1, p}(\Omega)$, it is not restrictive by density to assume that $U \in C_{c}^{\infty}(\Omega)$. Then, setting $U_{\varepsilon}\left(x_{i}\right):=U\left(x_{i}\right)$ with piecewise affine continuation, it is obvious that $U_{\varepsilon} \in B_{\phi}^{\varepsilon}$ and that $\nabla U_{\varepsilon} \rightarrow \nabla U$ uniformly as $\varepsilon \rightarrow 0$.

For $U \in C^{1}(\Omega) \cap \operatorname{Lip}_{\phi}(\bar{\Omega})$, we set again $U_{\varepsilon}\left(x_{i}\right):=U\left(x_{i}\right)$ with piecewise affine continuation, and define $V_{\varepsilon}:=$ $U_{\varepsilon}-U$. Since $U \in C(\bar{\Omega})$, we have $\left\|V_{\varepsilon}\right\|_{\infty} \rightarrow 0$, and thus $\left\|V_{\varepsilon}\right\|_{L^{p}(\Omega)} \rightarrow 0$. For the gradient, we split $\Omega=\Omega_{\delta} \cup N_{\delta}$, where the disjoint sets $\Omega_{\delta}$ and $N_{\delta}$ are such that $\overline{\Omega_{\delta}} \subset \Omega$ and $N_{\delta}$ has volume that vanishes as $\delta \rightarrow 0$. Then,

$$
\left\|\nabla V_{\varepsilon}\right\|_{L^{p}(\Omega)}^{p}=\left\|\nabla V_{\varepsilon}\right\|_{L^{p}\left(\Omega_{\delta}\right)}^{p}+\left\|\nabla V_{\varepsilon}\right\|_{L^{p}\left(N_{\delta}\right)}^{p} .
$$

First, since $\nabla U \in C\left(\overline{\Omega_{\delta}}\right)$, we have by the argument above that $\left\|\nabla V_{\varepsilon}\right\|_{C\left(\overline{\Omega_{\delta}}\right)} \rightarrow 0$, and thus $\left\|\nabla V_{\varepsilon}\right\|_{L^{p}\left(\Omega_{\delta}\right)}^{p} \rightarrow 0$ as $\varepsilon \rightarrow 0$. Second, since $U \in \operatorname{Lip}_{\phi}(\bar{\Omega}),\left\|\nabla U_{\varepsilon}\right\|_{\infty} \leq\|\nabla U\|_{\infty}<\infty$, and thus $\left\|\nabla V_{\varepsilon}\right\|_{L^{p}\left(N_{\delta}\right)}^{p} \leq C\left|N_{\delta}\right|$ uniformly in $\varepsilon$. Hence, by taking $\varepsilon$ small enough with respect to $\delta$ and $\delta \rightarrow 0$, we conclude that $\left\|\nabla V_{\varepsilon}\right\|_{L^{p}(\Omega)} \rightarrow 0$ as $\varepsilon \rightarrow 0$.

Thanks to Lemma 4.5, the proof of Proposition 4.4 narrows down to constructing a decomposition $U=$ $U_{1}+U_{2}+U_{3}$ where

$$
\left\{\begin{array}{l}
U_{1} \in C^{1}(\Omega) \cap \operatorname{Lip}_{\phi}(\bar{\Omega}), \\
U_{2} \in W_{0}^{1, p}(\Omega), \\
\left\|U_{3}\right\|_{W^{1, p}(\Omega)} \text { is small. }
\end{array}\right.
$$

Indeed, if such a decomposition exists, then Lemma 4.5 provides approximations in $B_{\phi}^{\varepsilon}$ of $U_{1}$ and $U_{2}$, and $U_{3}$ can simply be approximated by 0 . The difficulty in constructing such a decomposition is in finding a $U_{1}$ for which $\left.U_{1}\right|_{\Gamma}$ is sufficiently close to $\left.U\right|_{\Gamma}$. Approximation by convolution does not work directly since $U_{1}$ has to satisfy the boundary condition in $\operatorname{Lip}_{\phi}(\bar{\Omega})$. Instead, we use the Trace Theorem to approximate $\left.U\right|_{\Gamma}$ in an appropriate function space on $\Gamma$. This approximation is based on convolution, but care is needed because of the boundary condition, the corners of $\Gamma$ and the fact that the norm of the function space on $\Gamma$ is nonlocal.

Next, we prepare for proving Proposition 4.4 by citing a Trace Theorem (Lem. 4.6) and proving a density result on $\Gamma$ (Lem. 4.9). To avoid technical difficulties with the corners in $\Gamma$, we first transform $\Omega$ to the unit disc $\mathbb{D}=\left\{x \in \mathbb{R}^{2}:|x|<1\right\}$. With this aim, let $\varphi: \bar{\Omega} \rightarrow \overline{\mathbb{D}}$ be a related transformation (see Fig. 3 ) such that

$-\varphi$ is bi-Lipschitz;

$-\varphi \in C^{1}(\Omega)$

- $\varphi\left(\Gamma_{i}\right)=\gamma_{i}$ for $i=1,2,3$, where $\gamma_{i} \subset \partial \mathbb{D}$ are given, in terms of the polar angle coordinate, by

$$
\gamma_{1}:=\left[0, \frac{2 \pi}{3}\right], \quad \gamma_{2}:=\left[-\frac{2 \pi}{3}, 0\right], \quad \gamma_{3}:=\left[\frac{2 \pi}{3}, \frac{4 \pi}{3}\right] ;
$$

- if $U \in W_{\phi}^{1, p}(\Omega)$, then

$$
U \circ \varphi^{-1} \in W_{\phi}^{1, p}(\mathbb{D}):=\left\{V \in W^{1, p}(\mathbb{D}): V(-\theta)=R_{\phi} V(\theta) \text { for a.e. } 0<\theta<\frac{2 \pi}{3}\right\} .
$$

Above and in the following, we will often identify the unit circle $\mathbb{S}:=\partial \mathbb{D}$ with the periodic interval $[0,2 \pi)$. We also adopt the convention that a subscript $\phi$ in a function space indicates the boundary condition. We note 

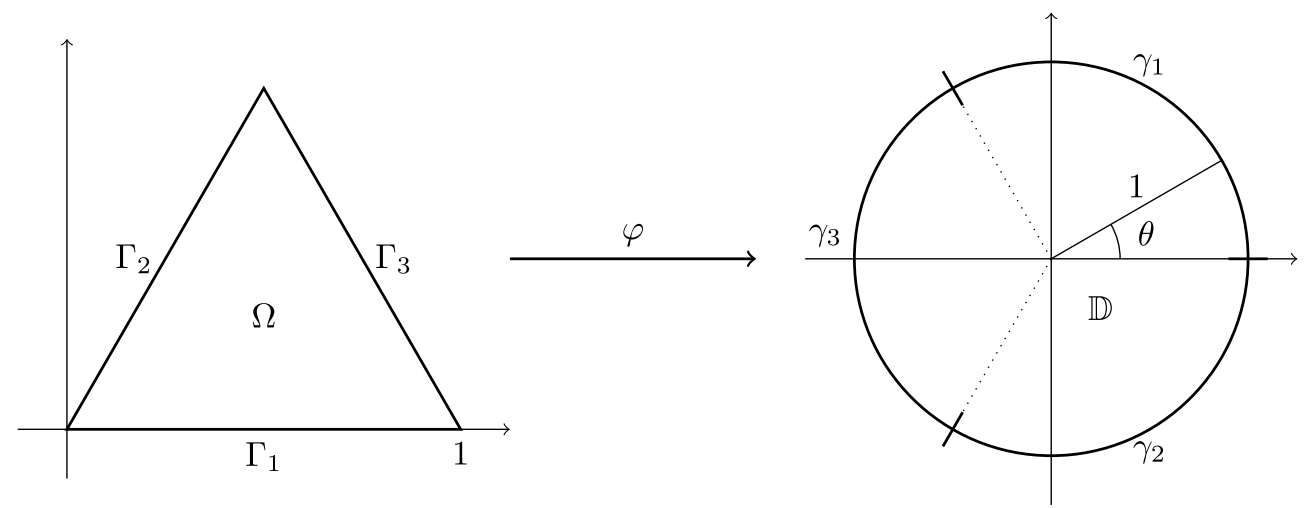

FiguRE 3 . The deformation $\varphi$ and the related sections of the boundaries of $\Omega$ and $\mathbb{D}$.

that there are constants $0<c, C$ such that for all $U \in W_{\phi}^{1, p}(\Omega)$

$$
c\|U\|_{W^{1, p}(\Omega)} \leq\left\|U \circ \varphi^{-1}\right\|_{W^{1, p}(\mathbb{D})} \leq C\|U\|_{W^{1, p}(\Omega)} .
$$

Hence, it is sufficient to construct the decomposition of $U$ after transforming it to $W_{\phi}^{1, p}(\mathbb{D})$.

To cite the Trace Theorem, we fix $s=1-\frac{1}{p}$ and recall the usual norm of the fractional Sobolev space $W^{s, p}(\mathbb{S})$ :

$$
\begin{aligned}
\|U\|_{W^{s, p}(\mathbb{S})}^{p} & :=\|U\|_{L^{p}(\mathbb{S})}^{p}+[U]_{W^{s, p}(\mathbb{S})}^{p}, \\
{[U]_{W^{s, p}(\mathbb{S})}^{p} } & :=\int_{0}^{2 \pi} \int_{0}^{2 \pi} \frac{|U(\theta)-U(\rho)|^{p}}{|\theta-\rho|_{\mathbb{S}}^{p}} d \rho d \theta, \quad|\theta|_{\mathbb{S}}=\min _{k \in \mathbb{Z}}|\theta-2 \pi k| .
\end{aligned}
$$

Lemma 4.6 (Trace $\left[24\right.$, Thm. 1.I]). There exists a $C>0$ such that for all $U \in W^{1, p}(\mathbb{D})$

$$
\left\|\left.U\right|_{\mathbb{S}}\right\|_{W^{s, p}(\mathbb{S})} \leq C\|U\|_{W^{1, p}(\mathbb{D})}
$$

Conversely, there exists a $C>0$ such that for all $f \in W^{s, p}(\mathbb{S})$ there exists a $U \in W^{1, p}(\mathbb{D})$ with $\left.U\right|_{\mathbb{S}}=f$ such that

$$
\|U\|_{W^{1, p}(\mathbb{D})} \leq C\|f\|_{W^{s, p}(\mathbb{S})}
$$

In order to prove a density result in $W_{\phi}^{s, p}(\mathbb{S})$, we first recall two technical lemmas:

Lemma 4.7 ([15], Lem. 5.2). If $f \in W^{s, p}(0, \pi)$, then the even extension $\tilde{f}$ satisfies

$$
\|\tilde{f}\|_{W^{s, p}(-\pi, \pi)} \leq C\|f\|_{W^{s, p}(0, \pi)}
$$

for some universal constant $C>0$.

Lemma 4.8 (Continuity of the translation operator). The translation operator $\tau_{a} f(\theta):=f(\theta+a)$ is continuous in the strong $W^{s, p}(\mathbb{S})$ topology, that is, for all $f \in W^{s, p}(\mathbb{S})$,

$$
\lim _{a \rightarrow 0}\left\|\tau_{a} f-f\right\|_{W^{s, p}(\mathbb{S})}=0 .
$$


Proof. The proof is standard; we give a sketch. The statement is obvious for $f \in C^{1}(\mathbb{S})$. For general $f \in W^{s, p}(\mathbb{S})$, it suffices to approximate it by $\psi \in C^{1}(\mathbb{S})$, and note that

$$
\left\|\tau_{a} \psi-\tau_{a} f\right\|_{W^{s, p}(\mathbb{S})}=\left\|\tau_{a}(\psi-f)\right\|_{W^{s, p}(\mathbb{S})}=\|\psi-f\|_{W^{s, p}(\mathbb{S})} .
$$

Lemma 4.9 (Approximation on $\mathbb{S}) . C_{\phi}^{\infty}(\mathbb{S})$ is dense in $W_{\phi}^{s, p}(\mathbb{S})$.

Proof. Let $f \in W_{\phi}^{s, p}(\mathbb{S})$. We split $f=f_{1}+f_{2}+f_{3}$ with $f_{i} \in W^{s, p}(0,2 \pi)$ such that

$$
f_{1}=\left\{\begin{array}{ll}
f & \text { on }\left(0, \frac{5 \pi}{6}\right) \\
0 & \text { on }\left(\frac{7 \pi}{6}, 2 \pi\right),
\end{array} f_{2}= \begin{cases}0 & \text { on }\left(0, \frac{5 \pi}{6}\right) \\
f & \text { on }\left(\frac{7 \pi}{6}, 2 \pi\right)\end{cases}\right.
$$

Note that $f_{3} \in W_{\phi}^{s, p}(\mathbb{S})$ with $\operatorname{supp} f_{3} \subset\left[\frac{5 \pi}{6}, \frac{7 \pi}{6}\right] \subset \subset \gamma_{3}$, and that it is not directly clear that $f_{1}, f_{2} \in W^{s, p}(\mathbb{S})$. We will construct approximating sequences $\left(\psi_{12}^{k}\right)_{k},\left(\psi_{3}^{k}\right)_{k} \subset C^{\infty}(\mathbb{S})$ of $f_{1}+f_{2}$ and $f_{3}$ respectively such that $\psi^{k}:=\psi_{12}^{k}+\psi_{3}^{k} \in C_{\phi}^{\infty}(\mathbb{S})$. By construction of $f_{3}, \psi_{3}^{k}:=\eta_{k} * f_{3}$ is a suitable approximating sequence, where $\eta_{k}$ is the usual mollifier.

To construct $\psi_{12}^{k}$, we first show that $f_{1}, f_{2} \in W^{s, p}(\mathbb{S})$. Since by construction $f_{1}, f_{2} \in W^{s, p}(0,2 \pi)$, it is sufficient to show that $f_{1}, f_{2} \in W^{s, p}(-r, r)$ for some $r>0$. We start with $f_{1}$. For any $\theta \in \mathbb{S}$ and any parameter $0 \leq \alpha \leq \pi$, let

$$
f_{1}^{\alpha}(\theta):= \begin{cases}R_{\alpha} f_{1}(-\theta) & -\pi<\theta<0 \\ f_{1}(\theta) & 0<\theta<\pi\end{cases}
$$

Clearly, $f_{1}^{\alpha} \in W^{s, p}(0, \pi) \cap W^{s, p}(\pi, 2 \pi)$ for any $\alpha$. Since $f_{1}^{\phi}(\theta)=f(\theta)$ for a.e. $\theta \in\left(-\frac{5 \pi}{6}, \frac{5 \pi}{6}\right)$ and $f \in W_{\phi}^{s, p}(\mathbb{S})$, we also have $f_{1}^{\phi} \in W^{s, p}\left(-\frac{5 \pi}{6}, \frac{5 \pi}{6}\right)$. Since $f_{1}^{0}$ is the even extension of $\left.f_{1}\right|_{(0, \pi)}$, we obtain from Lemma 4.7 that $f_{1}^{0} \in W^{s, p}(-\pi, \pi)$. Hence, for the odd extension $f_{1}^{\pi}$, we find from the linear relation

$$
f_{1}^{\phi}=\frac{I+R_{\phi}}{2} f_{1}^{0}+\frac{I-R_{\phi}}{2} f_{1}^{\pi}
$$

that $f_{1}^{\pi} \in W^{s, p}\left(-\frac{5 \pi}{6}, \frac{5 \pi}{6}\right)$. Finally,

$$
f_{1}=\frac{f_{1}^{0}+f_{1}^{\pi}}{2} \in W^{s, p}\left(-\frac{5 \pi}{6}, \frac{5 \pi}{6}\right)
$$

The proof of $f_{2} \in W^{s, p}\left(-\frac{5 \pi}{6}, \frac{5 \pi}{6}\right)$ is analogous.

Finally we construct the approximating sequences $\psi_{12}^{k}$. Care is needed to ensure that $\psi_{12}^{k}$ satisfies the boundary condition. With this aim, we first approximate $f_{1}$ and $f_{2}$ by the translations $\tau_{a} f_{1}$ and $\tau_{-a} f_{2}$ with $0<a<\frac{\pi}{6}$; see Lemma 4.8. Note that $\tau_{a} f_{1}+\tau_{-a} f_{2} \in W_{\phi}^{s, p}(\mathbb{S})$ and $\left.\left(\tau_{a} f_{1}+\tau_{-a} f_{2}\right)\right|_{(-a, a)} \equiv 0$. Then, we define $\psi_{12}^{k}:=\eta_{k} *\left(\tau_{a_{k}} f_{1}+\tau_{-a_{k}} f_{2}\right)$ for some $a_{k} \rightarrow 0$ such that supp $\eta_{k} \subset\left(-a_{k}, a_{k}\right)$, and note that $\psi_{12}^{k} \in C_{\phi}^{\infty}(\mathbb{S})$. This completes the proof.

We are ready to prove Proposition 4.4.

Proof of Proposition 4.4. Step 1: decomposition of $U$. Let $\delta>0$ and $U \in W_{\phi}^{1, p}(\Omega)$ be given. Set $\tilde{U}:=U \circ \varphi^{-1} \in$ $W_{\phi}^{1, p}(\mathbb{D})$ and $\tilde{f}:=\left.\tilde{U}\right|_{\mathbb{S}}$. Then, by Lemma 4.6 we have $\tilde{f} \in W_{\phi}^{s, p}(\mathbb{S})$. By Lemma 4.9 we find $\tilde{\psi} \in C_{\phi}^{\infty}(\mathbb{S})$ with $\|\tilde{\psi}-\tilde{f}\|_{W^{s, p}(\mathbb{S})}<\delta$. By Lemma 4.6, there exists $\tilde{U}_{3} \in W_{\phi}^{1, p}(\mathbb{D})$ with $\left.\tilde{U}_{3}\right|_{\mathbb{S}}=\tilde{f}-\tilde{\psi}$ and

$$
\left\|\tilde{U}_{3}\right\|_{W^{1, p}(\mathbb{D})} \leq C\|\tilde{\psi}-\tilde{f}\|_{W^{s, p}(\mathbb{S})} \leq C \delta
$$


for some $C>0$ independent of $\delta$.

Next, we take $\tilde{U}_{1} \in C_{\phi}^{\infty}(\mathbb{D})$ as the harmonic extension of $\tilde{\psi}$. Then, translating back to $\Omega$, we set $U_{i}:=\tilde{U}_{i} \circ \varphi$ for $i=1,3$. Taking $U_{2}:=U-U_{1}-U_{3}$, we observe that $U_{i}$ satisfy (4.4) for $i=1,2,3$.

Step 2: Construction of $U_{\varepsilon}$. By Lemma 4.5 we find sequences $\left(U_{1}^{\varepsilon}\right)_{\varepsilon},\left(U_{2}^{\varepsilon}\right)_{\varepsilon}$ parametrized by $\frac{1}{\varepsilon} \in \mathbb{N}$ such that $U_{i}^{\varepsilon} \in B_{\phi}^{\varepsilon}$ and

$$
\left\|U_{i}-U_{i}^{\varepsilon}\right\|_{W^{1, p}(\Omega)} \stackrel{\varepsilon \rightarrow 0}{\longrightarrow} 0
$$

for $i=1,2$. Hence, setting $U_{\varepsilon}:=U_{1}^{\varepsilon}+U_{2}^{\varepsilon}$ and taking $\varepsilon$ small enough with respect to $\delta$, we obtain

$$
\left\|U-U_{\varepsilon}\right\|_{W^{1, p}(\Omega)} \leq\left\|U_{1}-U_{1}^{\varepsilon}\right\|_{W^{1, p}(\Omega)}+\left\|U_{2}-U_{2}^{\varepsilon}\right\|_{W^{1, p}(\Omega)}+\left\|U_{3}\right\|_{W^{1, p}(\Omega)} \leq C \delta
$$

for some $C$ independent of $U_{i}, \delta$ or $\varepsilon$. Since $\delta>0$ is arbitrary, we conclude that $\left\|U-U_{\varepsilon}\right\|_{W^{1, p}(\Omega)} \rightarrow 0$ as $\varepsilon \rightarrow 0$.

\section{Physical interpretation of Theorem 3.1}

We show that, despite the relaxation process implied by $\Gamma$-convergence, the equilibrium solutions of the model are necessarily stressed. This is a consequence of the rotational boundary conditions incorporated into $W_{\phi}^{1, p}(\Omega)$ and of the finite penalization to folding imposed by $\Psi$.

\section{Proposition 5.1.}

$$
\min _{L^{p}(\Omega)} E>0
$$

The proof of Proposition 5.1 follows the lines of ([33], Sect. 5). For the readers' convenience, we display the main steps.

Proof. First, we show

$$
Q W(F) \geq c \operatorname{dist}^{p}(F, S O(2))
$$

for some $c>0$. From Lemma 4.3, we have $W(F) \geq c_{2} \operatorname{dist}^{p}(F, S O(2))$ for every $F \in \mathbb{R}^{2 \times 2}$. Now, thanks to (3.3), for every $\varepsilon>0$ and every smooth open $\omega \subset \mathbb{R}^{2}$ with $|\omega|=1$, there exists a map $\vartheta_{\varepsilon} \in W_{0}^{1, \infty}\left(\omega, \mathbb{R}^{2}\right)$ (in fact even $\left.C_{c}^{\infty}\left(\omega, \mathbb{R}^{2}\right)\right)$ such that

$$
Q W(F) \geq \int_{\omega} W\left(F+\nabla \vartheta_{\varepsilon}(x)\right) \mathrm{d} x-\varepsilon \geq c_{2} \int_{\omega} \operatorname{dist}^{p}\left(F+\nabla \vartheta_{\varepsilon}(x), S O(2)\right) \mathrm{d} x-\varepsilon .
$$

We now invoke Rigidity Theorem 3.1 [23] (which applies for $p \geq 2$, see ([12], Sect. 2.4)) yielding the existence of a constant $c>0$ and a matrix $R \in S O(2)$ such that

$$
c_{2} \int_{\omega} \operatorname{dist}^{p}\left(F+\nabla \vartheta_{\varepsilon}(x), S O(2)\right) \mathrm{d} x \geq c \int_{\omega}\left|F+\nabla \vartheta_{\varepsilon}(x)-R\right|^{p} \mathrm{~d} x .
$$

Now, interpreting $|\cdot|^{p}: \mathbb{R}^{2 \times 2} \rightarrow \mathbb{R}$ as a convex function on $\mathbb{R}^{4}$, we obtain

$$
\left|(F-R)+\nabla \vartheta_{\varepsilon}(x)\right|^{p} \geq|F-R|^{p}+p|F-R|^{p-2}(F-R): \nabla \vartheta_{\varepsilon}(x)
$$


for all $x \in \omega$. By applying (5.4) to (5.3) we have

$$
c_{2} \int_{\omega} \operatorname{dist}^{p}\left(F+\nabla \vartheta_{\varepsilon}, S O(2)\right) \mathrm{d} x \geq c \int_{\omega}|F-R|^{p} \mathrm{~d} x
$$

because the integral of $(F-R): \nabla \vartheta_{\varepsilon}$ vanishes as $\vartheta_{\varepsilon}$ has zero boundary datum. Putting our estimates together and minimising over $R$, we get

$$
Q W(F) \geq c \int_{\omega} \operatorname{dist}^{p}(F, S O(2)) \mathrm{d} x-\varepsilon
$$

and the desired result in (5.2) follows by sending $\varepsilon \rightarrow 0$.

Applying (5.2) we obtain

$$
\min _{W_{\phi}^{1, p}(\Omega)} E=E(\bar{U}) \geq c \int_{\Omega} \operatorname{dist}^{p}(\nabla \bar{U}, S O(2)) \mathrm{d} x .
$$

It is left to prove that the right-hand side is positive. Suppose instead that it is 0 . Then, $\nabla \bar{U} \in S O(2)$ a.e. in $\Omega$. Hence, $\nabla \bar{U}$ is a constant rotation (see [8]). This contradicts with $\bar{U} \in W_{\phi}^{1, p}(\Omega)$.

\section{NUMERICAL COMPUTATIONS}

In this section we explore numerically several energy wells of $E_{\varepsilon}$ and inspect whether the obtained minimizers satisfies $\operatorname{det} \nabla U_{\varepsilon}>0$. We do this for the simplified setting given by $\Psi \equiv 0$, which does not penalize folded patterns. Fortunately, this simplified setting turns out to be stable enough to reproduce configurations with $\operatorname{det} \nabla U_{\varepsilon}>0$ (see, e.g., Fig. 1) as local minima. Yet, local minimizers are of limited use to our theoretical result Theorem 3.1, because $\Gamma$-convergence only guarantees the convergence of global minimizers in the limit $\varepsilon \rightarrow 0$. Instead, we show by direct inspection of the energy values at the computed local minimizers that there is a good agreement with available continuum models for disclinations for decreasing values of $\varepsilon$. We compute local minimizers by employing Newton's method for different initial conditions. In all computations below, Newton's method converges quadratically.

\subsection{The limit $\varepsilon \rightarrow 0$}

Here we verify and quantify some of the computations in [42], in which disclinations satisfying $\operatorname{det} \nabla U_{\varepsilon}>0$ are obtained. The only difference with [42] is in the definition of $w$ in (2.4); in [42] $w \equiv 1$ is taken instead.

For both $\phi=\frac{2 \pi}{5}$ and $\phi=\frac{2 \pi}{7}$ we chose the initial condition such that the local minimizer of $E_{\varepsilon}$ satisfies $\operatorname{det} \nabla U_{\varepsilon}>0$. With this aim, for $\varepsilon=2^{-1}$ we took as initial condition one of the linear deformations $U_{\varepsilon}(x)=A x$ in $B_{\phi}^{\varepsilon}$ with $\operatorname{det} A=1$ (see Rem. 2.1). For the subsequent values $\varepsilon=2^{-2}, 2^{-3}, \ldots, 2^{-8}$, we constructed the initial condition from the minimizer obtained for the previous value of $\varepsilon$ by linear interpolation. The resulting local minimizers $\bar{U}_{\varepsilon}$ turn out to satisfy $\operatorname{det} \nabla \bar{U}_{\varepsilon}>0$. For $\varepsilon=2^{-3}, \bar{U}_{\varepsilon}$ is illustrated in Figure 1 . The energy values $e_{\varepsilon}:=E_{\varepsilon}\left(\bar{U}_{\varepsilon}\right)$ are plotted in Figure 4 . The behavior of $e_{\varepsilon}$ as $\varepsilon \rightarrow 0$ is qualitatively similar to the computations of [42] which are based on a model in linearized elasticity.

Next, we test the convergence of $e_{\varepsilon}$ as $\varepsilon \rightarrow 0$. Indeed, since $\bar{U}_{\varepsilon}$ need not be global minimizers, the $\Gamma$ convergence result in Theorem 3.1 does not guarantee convergence. To test the convergence, we impose the power law ansatz

$$
e_{\varepsilon} \sim C_{1}-C_{2} 2^{p}
$$




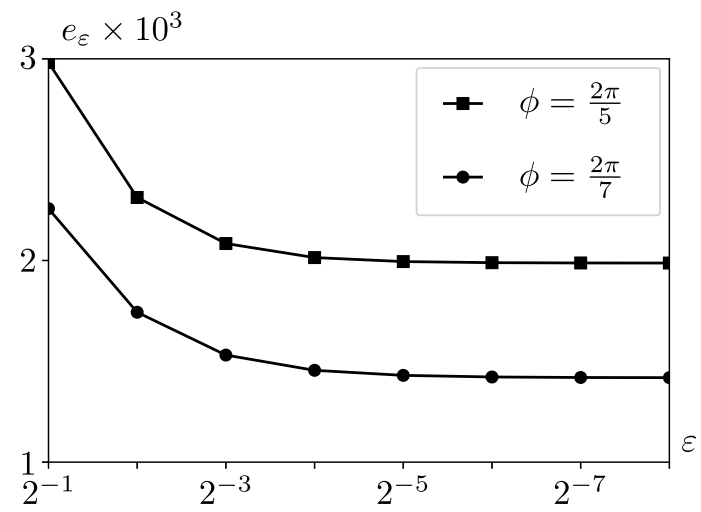

Figure 4 . The energy values $e_{\varepsilon}=E_{\varepsilon}\left(\bar{U}_{\varepsilon}\right)$ obtained from the simulations. Here, $\bar{U}_{\varepsilon}$ is verified to satisfy $\operatorname{det} \nabla \bar{U}_{\varepsilon}>0$.

TABLE 1 . The values of $p_{\varepsilon}$ (see (6.2) as computed from the numerical data to test the power law in the ansatz in (6.1)).

\begin{tabular}{ccc}
\hline & \multicolumn{2}{c}{$\phi$} \\
\cline { 2 - 3 }$\varepsilon$ & $\frac{2 \pi}{5}$ & $\frac{2 \pi}{7}$ \\
\hline $2^{-3}$ & 1.552 & 1.276 \\
$2^{-4}$ & 1.712 & 1.488 \\
$2^{-5}$ & 1.812 & 1.595 \\
$2^{-6}$ & 1.866 & 1.645 \\
$2^{-7}$ & 1.898 & 1.671 \\
$2^{-8}$ & 1.918 & 1.686 \\
\hline
\end{tabular}

where the constants $C_{1}, C_{2}, p>0$ need to be fitted from the data. Without computing $C_{1}$ and $C_{2}$, we obtain the exponent $p$ of the power law numerically from

$$
p_{\varepsilon}:=\frac{1}{\log 2} \log \frac{e_{4 \varepsilon}-e_{2 \varepsilon}}{e_{2 \varepsilon}-e_{\varepsilon}} .
$$

Table 1 lists the values of $p_{\varepsilon}$. Since the values of $p_{\varepsilon}$ are positive and increasing with $-\log \varepsilon, e_{\varepsilon}$ seems to converge as $\varepsilon \rightarrow 0$ implying a power law behavior.

\subsection{Folded configurations}

In the next simulations we explore other local minimizers of the energy by starting Newton's method from other initial conditions. In particular, since we take $\Psi \equiv 0$, there is no penalisation on $\operatorname{det} \nabla U_{\varepsilon} \leq 0$, and thus local minimizers with folded patterns may occur.

With this aim, we set $\varepsilon=2^{-2}$ and fold the reference lattice as illustrated in Figure 5 . This folding procedure is done such that the following property used in the boundary condition in (2.2)

$$
\Gamma_{2} \cap \mathcal{V}_{\varepsilon}=\left\{R_{\pi / 3} x: x \in \Gamma_{1} \cap \mathcal{V}_{\varepsilon}\right\}
$$

is conserved during the folding process. After folding, we construct the initial condition for Newton's method by deforming the folded reference domain by the linear map defined in Remark 2.1. 


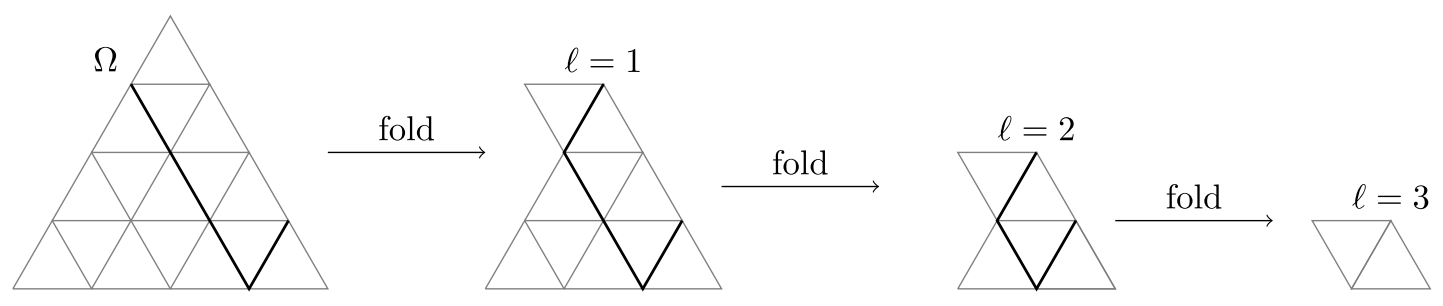

Figure 5. The folding procedure of the reference domain to construct the initial conditions. Lines along which we fold are drawn in boldface.
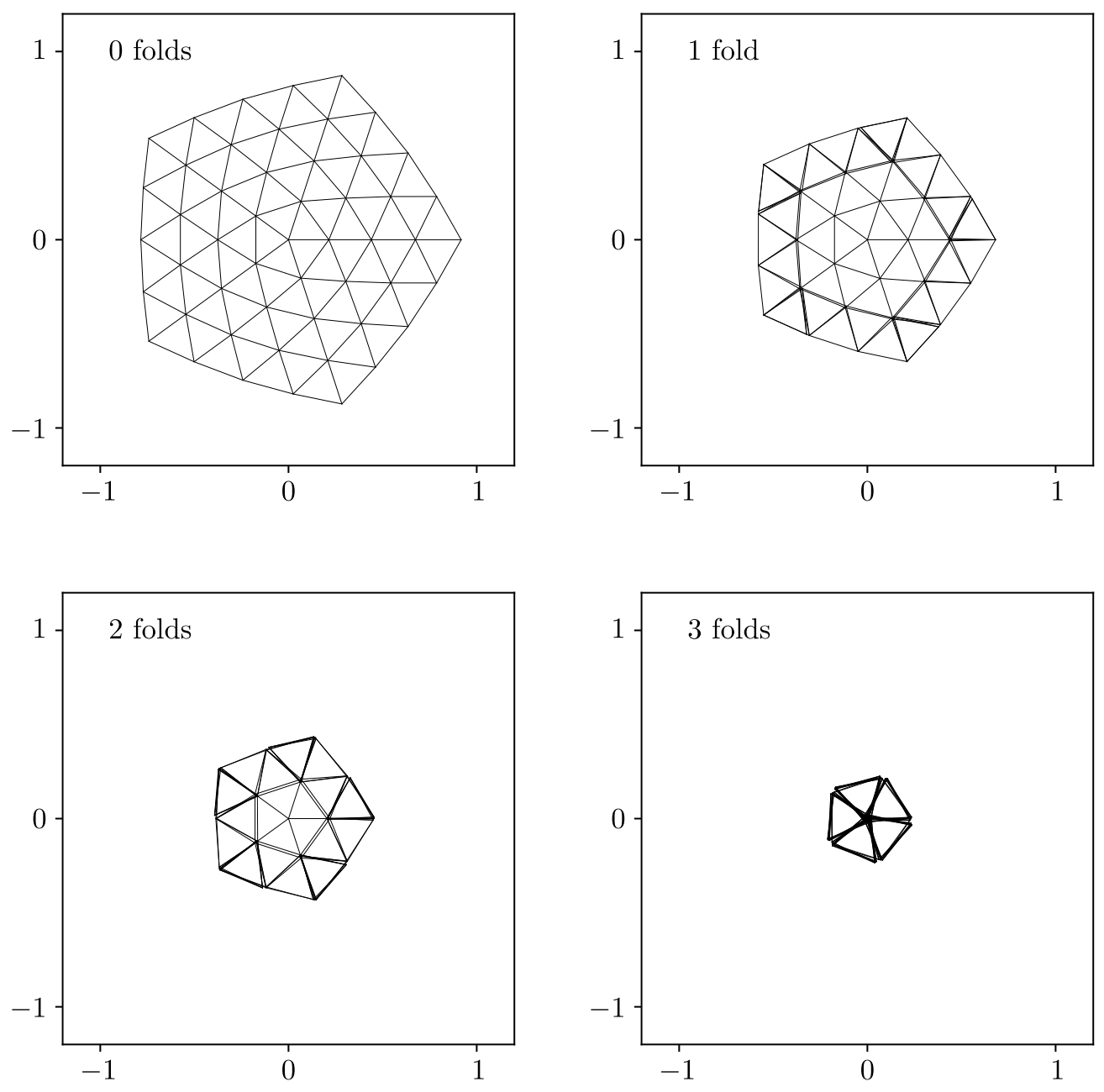

Figure 6. Minimizers of $E_{\varepsilon}$ obtained from the folded initial conditions shown in Figure 5.

Figures 6 and 7 illustrate the local minimizers obtained for each folded pattern shown in Figure 5 for $\phi=\frac{2 \pi}{5}$ and $\phi=\frac{2 \pi}{7}$ respectively. Figure 8 shows the related energy values. It is clear that the folded local minimizers have lower energy. In fact, it seems that the energy values decrease in an affine manner with the number of folds, and that, after linear extrapolation, 0 energy would be obtained around 4 folds. 

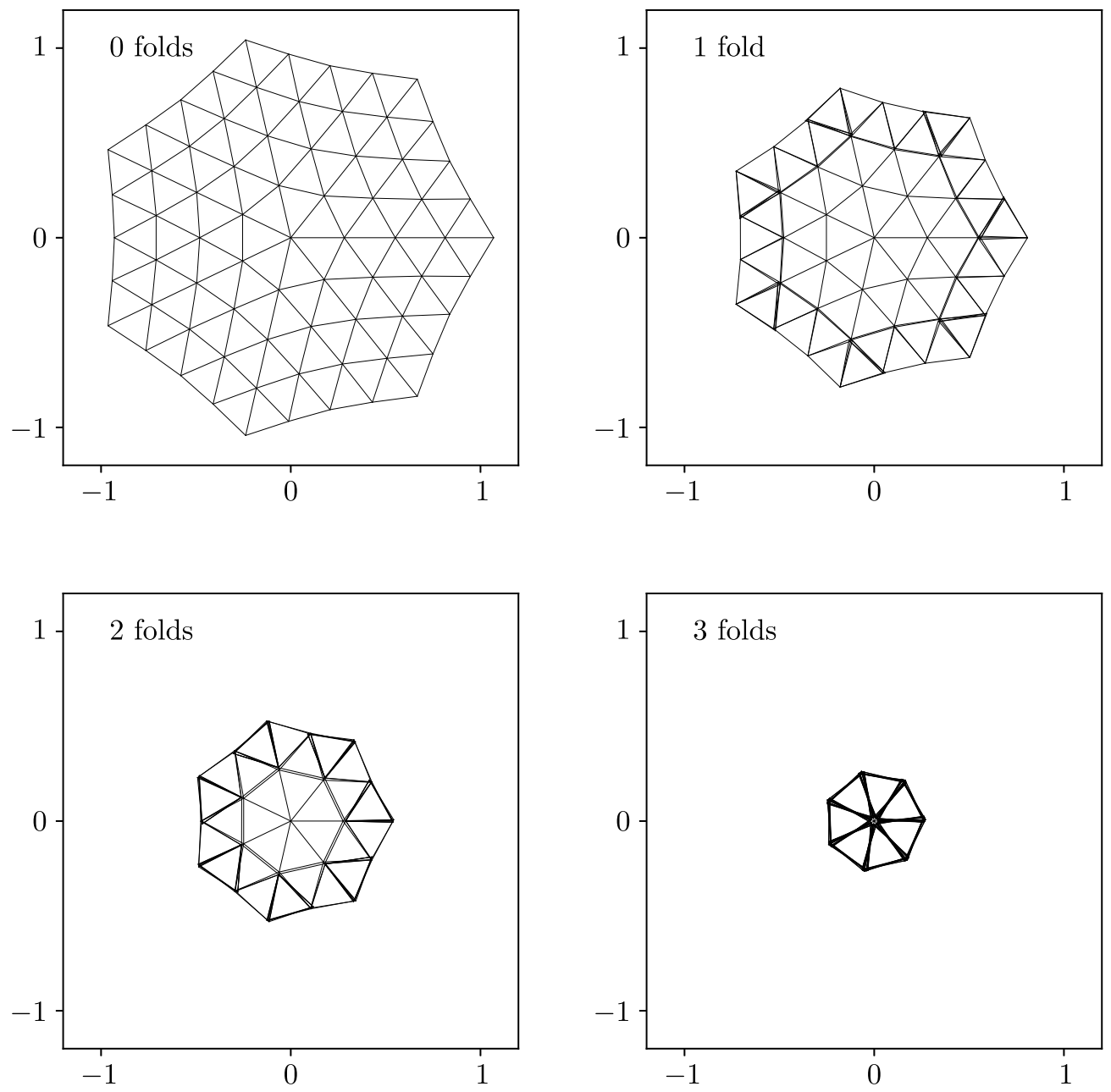

Figure 7. Minimizers of $E_{\varepsilon}$ obtained from the folded initial conditions shown in Figure 5.

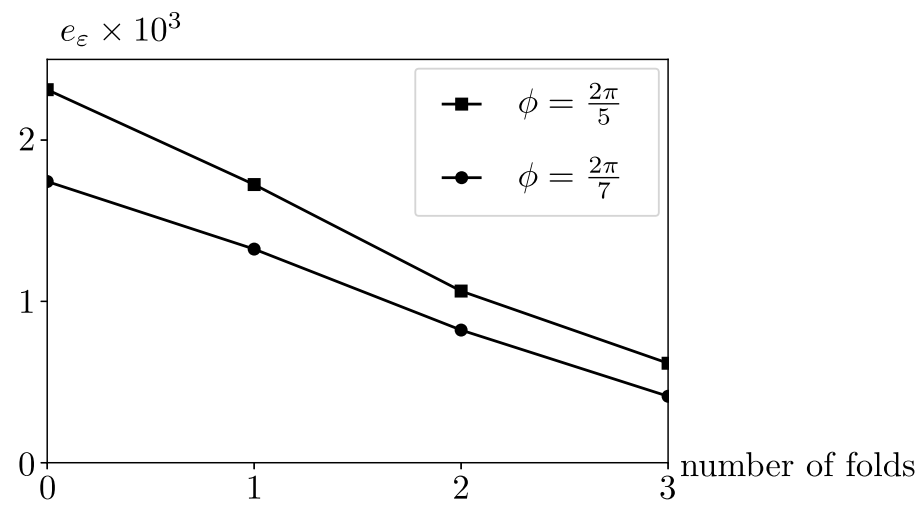

Figure 8 . The energy values $e_{\varepsilon}=E_{\varepsilon}\left(\bar{U}_{\varepsilon}\right)$ for $\varepsilon=2^{-2}$ computed from folded initial conditions (see Fig. 5). 
The observation that $E_{\varepsilon}$ has folded patterns as local minima with low energy is not unexpected from the expression of the continuum energy $E$. Indeed, $\Psi=0$ implies that the minimization problem of $E$ lacks rigidity; Lemma 4.3 and Proposition 5.1 are not valid anymore. In fact, if we substitute $\Psi=0$ into (2.8), we obtain (see Lemma A.2) $Q W(0)=0$, which implies that $0 \in L^{p}(\Omega)$ is a global minimizer of $E$.

This is consistent with the observation in Figure 8, where the energy values decay with the number of folds.

\section{Future PERSPECTIVES}

We develop a continuum model (the energy $E$ in (3.1)) for the energy of a disclination in a planar geometry. We construct $E$ rigorously from an atomistic model (Thm. 3.1), and show that any minimizer of $E$ corresponds to a stressed configuration of the medium (Prop. 5.1). We intend this first rigorous study as a stepping stone towards a general variational theory capable of describing both morphology and energetics of disclinations and their effect on the macrosocopic properties of a material. By analyzing the interaction of mismatches and distortions on the hexagonal lattice, we take a first step towards the investigation of the interaction of defects with the lattice kinematics, thus opening a possible path to modeling more complex systems such as austenite-martensite microstructures in Shape-Memory Alloys and kink formations as mentioned in the introduction.

\section{Appendix A.}

Lemma A.1. For all $0 \leq \sigma_{1} \leq \sigma_{2}$ and all $\theta \in \mathbb{R}$,

$$
14 \sum_{k=0}^{5}\left(\left|\Sigma R_{\theta+k \pi / 3} e_{1}\right|-1\right)^{2} \geq\left(\sigma_{1}-1\right)^{2}+\left(\sigma_{2}-1\right)^{2}, \quad \text { where } \Sigma:=\left[\begin{array}{cc}
\sigma_{1} & 0 \\
0 & \sigma_{2}
\end{array}\right] \text {. }
$$

Proof. We separate 2 cases: $\sigma_{1}^{2}+\sigma_{2}^{2} \geq 2$ and $\sigma_{1}^{2}+\sigma_{2}^{2} \leq 2$. In the first case, we start with bounding the right-hand side of (A.1) from above. If $\sigma_{1} \geq 1$, then clearly $\left(\sigma_{1}-1\right)^{2}+\left(\sigma_{2}-1\right)^{2} \leq 2\left(\sigma_{2}-1\right)^{2}$. If $\sigma_{1} \leq 1$, then from the observation that the line segment between the points $(0, \sqrt{2})$ and $(1,1)$ on the circle $\sqrt{2} \mathbb{S}$ is below the arc of $\sqrt{2} \mathbb{S}$ between the same two points, we obtain that $\sigma_{2} \geq 1+(\sqrt{2}-1)\left(1-\sigma_{1}\right)$. Hence,

$$
0 \leq 1-\sigma_{1} \leq \frac{\sigma_{2}-1}{\sqrt{2}-1}=(\sqrt{2}+1)\left(\sigma_{2}-1\right)
$$

This together with the bound for $\sigma_{1} \geq 1$ we get

$$
\left(\sigma_{1}-1\right)^{2}+\left(\sigma_{2}-1\right)^{2}<7\left(\sigma_{2}-1\right)^{2}
$$

We continue by bounding the left-hand side in (A.1) from below. Writing

$$
\left|\Sigma R_{\theta+k \pi / 3} e_{1}\right|^{2}=\sigma_{1}^{2} \cos ^{2}(\theta+k \pi / 3)+\sigma_{2}^{2} \sin ^{2}(\theta+k \pi / 3),
$$

we note from the facts that $\alpha \mapsto \sin ^{2} \alpha$ is $\pi$-periodic and $\left\{\alpha \in[0, \pi]: \sin ^{2} \alpha \geq \frac{3}{4}\right\}=[\pi / 3,2 \pi / 3]$ that there are at least two values for $k \in\{0, \ldots, 5\}$ for which $\sin ^{2}(\theta+k \pi / 3) \geq \frac{3}{4}$. For these values of $k$, we have by $\sigma_{2} \geq \max \left\{1, \sigma_{1}\right\}$ and $\sigma_{1}^{2}+\sigma_{2}^{2} \geq 2$ that

$$
\left|\Sigma R_{\theta+k \pi / 3} e_{1}\right|^{2} \geq \frac{1}{4} \sigma_{1}^{2}+\frac{3}{4} \sigma_{2}^{2} \geq \frac{1}{2} \sigma_{2}^{2}+\frac{1}{2} \geq 1,
$$


and thus

$$
14 \sum_{k=0}^{5}\left(\left|\Sigma R_{\theta+k \pi / 3} e_{1}\right|-1\right)^{2} \geq 28\left(\frac{1}{\sqrt{2}} \sqrt{\sigma_{2}^{2}+1}-1\right)^{2} .
$$

Estimating the convex function $f(x)=\sqrt{x^{2}+1}$ from below by its tangent at $x=1$ yields

$$
\sqrt{\sigma_{2}^{2}+1} \geq \sqrt{2}+\left(\sigma_{2}-1\right) / \sqrt{2}
$$

Plugging this estimate into (A.3), we observe that the resulting lower bound equals the upper bound in (A.2). This completes the proof in the case $\sigma_{1}^{2}+\sigma_{2}^{2} \geq 2$.

In the second case, $\sigma_{1}^{2}+\sigma_{2}^{2} \leq 2$, we follow a similar procedure. We claim that $\left(\sigma_{2}-1\right)^{2} \leq\left(\sigma_{1}-1\right)^{2}$. Then, instead of (A.2), we get

$$
\left(\sigma_{1}-1\right)^{2}+\left(\sigma_{2}-1\right)^{2} \leq 2\left(1-\sigma_{1}\right)^{2}
$$

To prove this claim, we note from $\sigma_{1} \leq \sigma_{2}$ that $\sigma_{1}-1 \leq \sigma_{2}-1$. To get an upper bound for $\sigma_{2}-1$, we obtain from $\sigma_{1}^{2}+\sigma_{2}^{2} \leq 2$ that

$$
\sigma_{2} \leq \sqrt{2-\sigma_{1}^{2}}
$$

Since the right-hand side is concave in $\sigma_{1}$, we can bound it from above by its tangent at $\sigma_{1}=1$, this yields $\sigma_{2} \leq 2-\sigma_{1}$, and thus $\sigma_{2}-1 \leq 1-\sigma_{1}$. The claim follows.

For the left-hand side of (A.1), similar to the previous case, there are at least two values for $k \in\{0, \ldots, 5\}$ for which $\cos ^{2}(\theta+k \pi / 3) \geq \frac{3}{4}$. For these values of $k$, we have by $\sigma_{1} \leq \sigma_{2}, \sigma_{1}^{2}+\sigma_{2}^{2} \leq 2$ and $\sigma_{1} \leq 1$ that

$$
\left|\Sigma R_{\theta+k \pi / 3} e_{1}\right|^{2} \leq \frac{3}{4} \sigma_{1}^{2}+\frac{1}{4} \sigma_{2}^{2} \leq \frac{1}{2} \sigma_{1}^{2}+\frac{1}{2} \leq 1,
$$

and thus

$$
14 \sum_{k=0}^{5}\left(1-\left|\Sigma R_{\theta+k \pi / 3} e_{1}\right|\right)^{2} \geq 28\left(1-\frac{1}{\sqrt{2}} \sqrt{\sigma_{1}^{2}+1}\right)^{2} .
$$

Using that $f(x)=\sqrt{x^{2}+1} \leq f(1)+(1-x)(f(0)-f(1))$, we obtain

$$
\sqrt{\sigma_{1}^{2}+1} \leq \sqrt{2}-\left(1-\sigma_{1}\right)(\sqrt{2}-1)
$$

Plugging this estimate into (A.5), we observe that the resulting lower bound is larger than the upper bound in (A.4).

Lemma A.2. Let $W$ be as in (2.8) with $\Psi=0$, that is,

$$
W(A)=\sum_{e \in B^{1}} \Phi(|e A|-1)
$$


Then,

$$
Q W(0)=0
$$

Proof. Since $0 \leq W(A)$ for all $A \in \mathbb{R}^{2 \times 2}$, it follows $0 \leq Q W(0)$. We are left to show the reverse inequality. To do this, we introduce $R W: \mathbb{R}^{2} \rightarrow \mathbb{R}$, the rank-1 convex envelope of $W$ (see [13], Sect. 6.4) which is characterized by the following formula ([13], Thm. 6.10)

$$
R W(A)=\inf \left\{\sum_{i=1}^{I} \lambda_{i} W\left(A_{i}\right): I \in \mathbb{N}, \lambda_{i} \geq 0, \sum_{i=1}^{I} \lambda_{i}=1, \sum_{i=1}^{I} \lambda_{i} A_{i}=A,\left(\lambda_{i}, A_{i}\right) \text { satisfy }\left(H_{I}\right)\right\},
$$

where $\left(H_{I}\right)$ is the hierarchical compatibility constraint defined in [13], Definition 5.14. Recall also that for $W: \mathbb{R}^{2} \rightarrow \mathbb{R}$, then $Q W \leq R W$ (see [13], Sect. 6.1). Hence, it remains to show that $R W(0) \leq 0$.

To show that $R W(0) \leq 0$, we take in (A.6) $I=4, \lambda_{i}=\frac{1}{4}$ and consider the family

$$
A_{1}:=\left[\begin{array}{cc}
1 & 0 \\
0 & -1
\end{array}\right], A_{2}:=\left[\begin{array}{ll}
1 & 0 \\
0 & 1
\end{array}\right], A_{3}=-A_{1}, A_{4}=-A_{2}
$$

and observe that

1) $\frac{1}{4} A_{1}+\frac{1}{4} A_{2}+\frac{1}{4} A_{3}+\frac{1}{4} A_{4}=0$

2) $\operatorname{rank}\left(A_{1}-A_{2}\right)=\operatorname{rank}\left(A_{3}-A_{4}\right)=1$;

3) $\operatorname{rank}\left(\frac{A_{1}+A_{2}}{2}-\frac{A_{3}+A_{4}}{2}\right)=1$;

4) $\left|A_{i} e_{j}\right|^{2}=e_{j}^{T} A_{i}^{T} A_{i} e_{j}=1, j=1, \ldots, 6$ where $e_{j} \in B^{1}$ and $i=1, \ldots, 4$.

By Example 5.15 of [13], Properties (2)-(3) imply that $\left(\lambda_{i}, A_{i}\right)$ satisfy condition $\left(H_{I}\right)$. Then, it is then easy to see from (1) that $\left(\lambda_{i}, A_{i}\right)$ constitute an admissible candidate in the minimization problem (A.6). Finally, condition (4) implies $W\left(A_{i}\right)=0$ for $i=1, \ldots, 4$.

Collecting all the results above, we have

$$
0 \leq Q W(0) \leq R W(0) \leq \sum_{i=1}^{4} \lambda_{i} W\left(A_{i}\right)=0 .
$$

Acknowledgements. P.C. is supported by by JSPS Innovative Area Grant JP19H05131. P.C. holds an honorary appointment at La Trobe University and is a member of GNAMPA. P.vM. gratefully acknowledges support from JSPS KAKENHI Grant Number JP20K14358. The authors acknowledge the Research Institute for Mathematical Sciences, an International Joint Usage and Research Center located in Kyoto University, where part of the work contained in this paper was carried out.

\section{REFERENCES}

[1] K. Anthony, U. Essmann, A. Seeger and H. Trauble, Disclinations and the Cosserat-Continuum with Incompatible Rotations, volume Mechanics of Generalized Continua, Proceedings of the IUTAM-Symposium on The Generalized Cosserat Continuum and the Continuum Theory of Dislocations with Applications, Freudenstadt and Stuttgart (Germany), 1967. Springer-Verlag Berlin Heidelberg (1968) 355-358.

[2] E. Acerbi and N. Fusco, Semicontinuity problems in the calculus of variations. Arch. Ratl. Mech. Anal. 86 (1984) $125-145$.

[3] M.P. Ariza and M. Ortiz, Discrete crystal elasticity and discrete dislocations in crystals. Arch. Ratl. Mech. Anal. 178 (2005) $149-226$.

[4] J. Braun, M. Buze and C. Ortner, The effect of crystal symmetries on the locality of screw dislocation cores. SIAM J. Math. Anal. 51 (2019) 1108-1136.

[5] J. Ball, P. Cesana and P. Hambly, A probabilistic model for martensitic avalanches. MATEC Web Conf. 33 (2015) 1-6. 
[6] K. Bhattacharya, Microstructure of martensite: why it forms and how it gives rise to the shape-memory effect. Oxford University Press (2003).

[7] M. Buze, T. Hudson and C. Ortner, Analysis of an atomistic model for anti-plane fracture. Math. Models Methods Appl. Sci. 29 (2019) 2469-2521.

[8] J. Ball and R. James, Fine phase mixtures as minimizers of eenergies. Arch. Ration. Mech. Anal. 100 (1987) $13-52$.

[9] P. Cesana, F. Della Porta, A. Rueland, C. Zillinger and B. Zwicknagl, Exact constructions in the (non-linear) planar theory of elasticity: from elastic crystals to nematic elastomers. Arch. Ratl. Mech. Anal. 237 (2020) 383-445.

[10] P. Cesana and P. Hambly, A probabilistic model for interfaces in a martensitic phase transition. Preprint https://arxiv.org/ abs/1810.04380 (2018).

[11] P. Cesana, M. Porta and T. Lookman, Asymptotic analysis of hierarchical martensitic microstructure. J. Mech. Phys. Solids 72 (2014) 174-192.

[12] S. Conti and B. Schweizer, Rigidity and gamma convergence for solid-solid phase transitions with $\mathrm{SO}(2)$ invariance. Commun. Pure Appl. Math. 59 (2006) 830-868.

[13] B. Dacorogna, Direct Methods in the Calculus of Variations. Springer, Heidelberg, 2nd edition (2008).

[14] G. Dal Maso, An Introduction to Г-Convergence. Birkhäuser Verlag, Boston (1993).

[15] E. Di Nezza, G. Palatucci and E. Valdinoci, Hitchhiker's guide to the fractional Sobolev spaces. Bull. Sci. Math. 136 (2012) $521-573$.

[16] R. de Wit, Linear theory of static disclinations. Vol. 317 of Fundamental Aspects of Dislocation Theory, edited by J.A. Simmons, R. de Wit, and R. Bullough. Nat. Bur. Stand. (US), Spec. Publ. (1970) 651-673.

[17] R. de Wit, Theory of disclinations: II. continuous and discrete disclinations in anisotropic elasticity. J. Res. Natl. Bureau Stand. A 77A (1973).

[18] R. de Wit, Theory of disclinations: III. continuous and discrete disclinations in isotropic elasticity. J. Res. Natl. Bureau Stand. A 73A (1973).

[19] R. de Wit, Theory of disclinations: IV. straight disclinations. J. Res. Natl. Bureau Stand. A 77A (1973).

[20] V. Ehrlacher, C. Ortner and A.V. Shapeev, Analysis of boundary conditions for crystal defect atomistic simulations. Arch. Ratl. Mech. Anal. 222 (2016) 1217-1268.

[21] I. Ekeland and R. Temam, Vol. 28 of Convex Analysis and Variational Problems. SIAM (1999).

[22] D. Essmann and H. Träuble, The direct observation of individual flux lines in type ii superconductors. Phys. Lett. 24A (1967).

[23] G. Friesecke, R. James and S. Müller, A theorem on geometric rigidity and the derivation of non linear plate theory from three dimensional elasticity. Commun. Pure Appl. Math. 55 (2002) 1461-1506.

[24] E. Gagliardo, Caratterizzazioni delle tracce sulla frontiera relative ad alcune classi di funzioni in n variabili. Rend. Sem. Mat. Univ. Padova 27 (1957) 284-305.

[25] K. Hagihara, T. Mayama, M. Honnami, M. Yamasaki, H. Izuno, T. Okamoto, T. Ohashi, T. Nakano and Y. Kawamura, Orientation dependence of the deformation kink band formation behavior in Zn single crystal. Int. J. Plasticity $\mathbf{7 7}$ (2016) $174-191$.

[26] K. Hagihara, T. Okamoto, H. Izuno, M. Yamasaki, M. Matsushita, T. Nakano and Y. Kawamura, Plastic deformation behavior of 10H-type synchronized LPSO phase in a Mg-Zn-Y system. Acta Mater. 109 (2016) 90-102.

[27] T. Hudson and C. Ortner, Existence and stability of a screw dislocation under anti-plane deformation. Arch. Ratl. Mech. Anal. 213 (2014) 887-929.

[28] T. Hudson and C. Ortner, Analysis of stable screw dislocation configurations in an antiplane lattice model. SIAM J. Math. Anal. 47 (2015) 291-320.

[29] T. Inamura, H. Hosoda and S. Miyazaki, Incompatibility and preferred morphology in the self-accommodation microstructure of $\beta$-titanium shape memory alloy. Philos. Mag. 93 (2013) 618-634.

[30] T. Inamura, M. Li, M. Tahara and H. Hosoda, Formation process of the incompatible martensite microstructure in a betatitanium shape memory alloy. Acta Mater. 124 (2017) 351-359.

[31] T. Inamura, Geometry of kink microstructure analysed by rank-1 connection. Acta Mater. 173 (2019) 270-280.

[32] Y. Kitano and K. Kifune, HREM study of disclinations in MgCd ordered alloy. Ultramicroscopy 39 (1991) $279-286$.

[33] R. Kupferman and C. Maor, Variational convergence of discrete geometrically-incompatible elastic models. Calc. Var. Partial Differ. Equ. 57 (2018).

[34] M. Lazar, Wedge disclination in the field theory of elastoplasticity. Phys. Lett. A 311 (2003) 416-425.

[35] G. Lazzaroni, M. Palombaro and A. Schlömerkemper, A discrete to continuum analysis of dislocations in nanowire heterostructures. Commun. Math. Sci. 13 (2015) 1105-1133.

[36] X.W. Lei and A. Nakatani, A deformation mechanism for ridge-shaped kink structure in layered solids. J. Appl. Mech. 82 (2015) 071016.

[37] C. Manolikas and S. Amelinckx, Phase transitions in ferroelastic lead orthovanadate as observed by means of electron microscopy and electron diffraction. Phys. Stat. Sol. 60 (1980) 607-617.

[38] F.R.N. Nabarro, Theory of crystal dislocations. International Series of Monographs on Physics. Oxford: Clarendon Press (1967).

[39] M. Porta and T. Lookman, Heterogeneity and phase transformation in materials: energy minimization, iterative methods and geometric nonlinearity. Acta Mater. 61 (2013) 5311-5340. 
[40] M. Ponsiglione, Elastic energy stored in a crystal induced by screw dislocations: from discrete to continuous. SIAM J. Math. Anal. 39 (2007) 449-469.

[41] A.E. Romanov and V.I. Vladimirov, in Vol. 9 of Dislocations in solids, edited by F.R.N. Nabarro. North-Holland, Amsterdam (1992) 191

[42] S. Seung, and R. Nelson, Defects in flexible membranes with crystalline order. Phys. Rev. A 38 (1988) 1005.

[43] H. Träuble and D. Essmann, Fehler im flussliniengitter von supraleitern zweiter art. Phys. Stat. Sol. 25 (1968).

[44] V. Volterra, Sur l'équilibre des corps élastiques multiplement connexes. Ann. scientifiques de l'École Normale Supérieure $\mathbf{2 4}$ (1907) 401-517.

[45] C. Zhang and A. Acharya, On the relevance of generalized disclinations in defect mechanics. J. Mech. Phys. Solids 119 (2018) $188-223$.

[46] L.M. Zubov, Nonlinear Theory of Dislocations and Disclinations in Elastic Bodies. Lecture Notes in Physics Monographs. Springer (1997) 